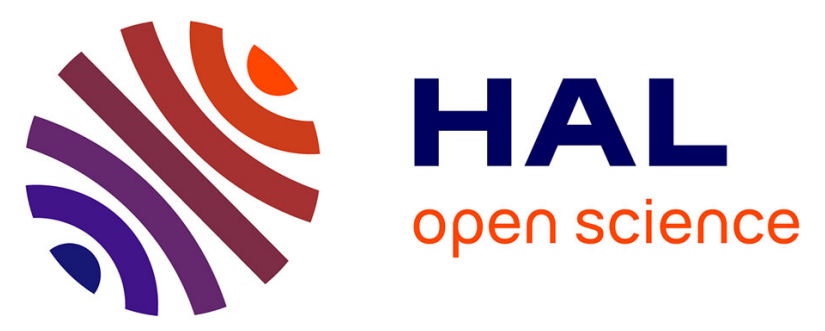

\title{
Switching between Coherent and Incoherent Singlet Fission via Solvent-Induced Symmetry Breaking
}

Antonios M Alvertis, Steven Lukman, Timothy J H Hele, Eric G Fuemmeler, Jiaqi Feng, Jishan Wu, Neil C Greenham, Alex W Chin, Andrew J Musser

\section{To cite this version:}

Antonios M Alvertis, Steven Lukman, Timothy J H Hele, Eric G Fuemmeler, Jiaqi Feng, et al.. Switching between Coherent and Incoherent Singlet Fission via Solvent-Induced Symmetry Breaking. Journal of the American Chemical Society, 2019, 141 (44), pp.17558-17570. 10.1021/jacs.9b05561 . hal-02377577

\section{HAL Id: hal-02377577 \\ https://hal.sorbonne-universite.fr/hal-02377577}

Submitted on 15 Dec 2021

HAL is a multi-disciplinary open access archive for the deposit and dissemination of scientific research documents, whether they are published or not. The documents may come from teaching and research institutions in France or abroad, or from public or private research centers.
L'archive ouverte pluridisciplinaire HAL, est destinée au dépôt et à la diffusion de documents scientifiques de niveau recherche, publiés ou non, émanant des établissements d'enseignement et de recherche français ou étrangers, des laboratoires publics ou privés. 


\section{Article}

\section{Switching between coherent and incoherent singlet fission via solvent-induced symmetry-breaking}

Antonios M Alvertis, Steven Lukman, Timothy J. H. Hele, Eric G Fuemmeler, Jiaqi Feng, Jishan Wu, Neil C. Greenham, Alex W Chin, and Andrew J. Musser

J. Am. Chem. Soc., Just Accepted Manuscript • DOI: 10.1021/jacs.9b05561 • Publication Date (Web): 11 Oct 2019

Downloaded from pubs.acs.org on October 23, 2019

\section{Just Accepted}

"Just Accepted" manuscripts have been peer-reviewed and accepted for publication. They are posted online prior to technical editing, formatting for publication and author proofing. The American Chemical Society provides "Just Accepted" as a service to the research community to expedite the dissemination of scientific material as soon as possible after acceptance. "Just Accepted" manuscripts appear in full in PDF format accompanied by an HTML abstract. "Just Accepted" manuscripts have been fully peer reviewed, but should not be considered the official version of record. They are citable by the Digital Object Identifier (DOI®). "Just Accepted" is an optional service offered to authors. Therefore, the "Just Accepted" Web site may not include all articles that will be published in the journal. After a manuscript is technically edited and formatted, it will be removed from the "Just Accepted" Web site and published as an ASAP article. Note that technical editing may introduce minor changes to the manuscript text and/or graphics which could affect content, and all legal disclaimers and ethical guidelines that apply to the journal pertain. ACS cannot be held responsible for errors or consequences arising from the use of information contained in these "Just Accepted" manuscripts. 


\title{
Switching between coherent and incoherent singlet fission via
}

\section{solvent-induced symmetry-breaking}

\author{
Antonios M. Alvertis ${ }^{1, \dagger}$, Steven Lukman ${ }^{2, \dagger}$, Timothy J. H. Hele ${ }^{1}$, Eric G. Fuemmeler ${ }^{3}$, Jiaqi Feng ${ }^{4}$, Jishan \\ $\mathrm{Wu}^{4}$, Neil C. Greenham ${ }^{1}$, Alex W. Chin ${ }^{5}$, Andrew J. Musser ${ }^{3,6, *}$ \\ ${ }^{1}$ Cavendish Laboratory, University of Cambridge, J. J. Thomson Avenue, Cambridge CB3 0HE, United Kingdom \\ ${ }^{2}$ Institute of Materials Research and Engineering, Agency for Science Technology and Research (A*STAR), 2 Fusionopolis Way, \\ Singapore 138634, Singapore. \\ ${ }^{3}$ Department of Chemistry and Chemical Biology, Cornell University, Ithaca, NY 14853 \\ ${ }^{4}$ Department of Chemistry, National University of Singapore, 3 Science Drive 3, 117543, Singapore \\ ${ }^{5}$ CNRS \& Institut des NanoSciences de Paris, Sorbonne Université, 4 place Jussieu boite courrier 840,75252 Paris Cedex 05, \\ France. \\ ${ }^{6}$ Department of Physics and Astronomy, University of Sheffield, Hounsfield Road, Sheffield S3 7RH, UK. \\ $\dagger^{\dagger}$ These authors contributed equally. \\ *e-mail: ajm557@cornell.edu
}

\begin{abstract}
Singlet fission in organic semiconductors causes a singlet exciton to decay into a pair of triplet excitons and holds potential for increasing the efficiency of photovoltaic devices. In this combined experimental and theoretical study, we reveal that a covalent dimer of the organic semiconductor tetracene undergoes activated singlet fission by qualitatively different mechanisms depending on the solvent environment. We show that intramolecular vibrations are an integral part of this mechanism, giving rise to mixing between charge transfer and triplet pair excitations. Both coherent or incoherent singlet fission can occur, depending on transient solvent-induced energetic proximity between the states, giving rise to complex variation of the singlet fission mechanism and timescale in the different environments. Our results suggest a more general principle for controlling the efficiency of photochemical reactions by utilizing transient interactions to tune the energetics of reactant and product states and switch between incoherent and coherent dynamics.
\end{abstract}




\section{Introduction}

Singlet fission 1 is an electronic process in organic materials which has been extensively studied in the past decade. This is largely because of its promise for efficient solar energy technologies which surpass the ShockleyQueisser limit!2 Singlet fission converts a high-energy singlet exciton to two low-energy triplet excitons, which are at least initially coupled into an overall singlet state. Thus singlet fission conserves spin, allowing it to be an ultra-fast process and effectively compete with radiative and non-radiative deactivation of excited states. This energetic down-conversion process offers a way to overcome thermalization losses, and has inspired the design of new hybrid device concepts $\sqrt[3]{3}$ However, the primary interest in the field to date remains building a more detailed understanding of the underlying photophysical mechanism, with the aim of informing rational materials design.

There have been multiple reports that singlet fission can occur through an ultrafast (fs-ps) 'coherent' mechanism. $5+9]$ Here, photoexcitation is thought to generate an initial superposition of the lowest bright singlet state $\mathrm{S}_{1}$ and the dark double-triplet state TT which eventually dephases into the dark state. In some materials, this coherent regime is reported to coexist with slower, incoherent fission dynamics,$\left[\sqrt[8]{9}\right.$ in which $\mathrm{S}_{1}$ 'hops' to the TT surface non-adiabatically, or adiabatically relaxes into the TT region on the same potential energy surface. Incoherent singlet fission is the more commonly invoked picture, particularly when it occurs on longer ( $>$ ps) timescales $\frac{10[11}{10}$ Interestingly, even in systems where it is endothermic, e.g. in tetracene, $\frac{12}{15}$ singlet fission can be very efficient and temperature-independent 14 17 To explain such observations, coherent processes have been invoked $9\left[\begin{array}{ll}9 & 18\end{array}\right.$

Despite recent progress in understanding the coherent mechanism of singlet fission and its interplay with incoherent dynamics, what has thus far been missing is a tuneable way of switching between the two regimes. This is partly because there are no established experimental handles to achieve this, and the utility of coherent dynamics as a concept for materials design and eventual applications is not clear. Similarly to many other photophysical processes in organic molecules, one of the most promising angles to explore coherent dynamics is

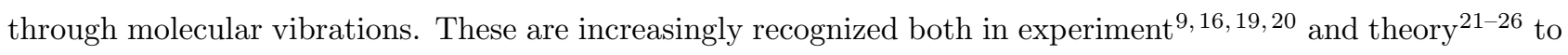
play a critical role in the ultra-fast regime where singlet fission is often found to occur. The coherent mechanism of singlet fission in rubrene (which has a well-defined symmetry) has been shown to arise via symmetry-breaking modes that allow $\mathrm{S}_{1}$ and TT to mix $!^{9}$ More broadly, when the mixing between electronic states in such systems is governed by coupling to intramolecular motions, to some extent it can be tuned, for example by controlling the viscosity of the environment ${ }^{27}$ or chemically introducing steric barriers. ${ }^{28}$ This control is rather limited and poorly understood, especially in solid-state systems where the complex interplay of intra- and intermolecular vibrations must be considered.

Another critical ingredient in both coherent and incoherent fission mechanisms that has proved most difficult to explore is the nature of the coupling that results in quantum superpositions or population transfer between $\mathrm{S}_{1}$ and TT. There has been extensive debate about the relative strength and influence of 'direct' two-electron coupling between these states, versus sequential one-electron couplings mediated by a charge-transfer (CT) 
state $1 \sqrt[11]{29} \sqrt[32]{32}$ Couplings involving the CT state are typically expected to be orders of magnitude higher, but in conventional thin-film systems there is no experimental means to perturb the CT manifold, especially if the states are not directly populated but only mediate fission through super-exchange as 'virtual' intermediates 2,29 However, covalent dimers of singlet-fission chromophores offer exquisite tunability of the critical interactions that govern fission, both through chemical design $\sqrt{33} \sqrt[43]{3}$ and the external environment, $27 \sqrt[44]{45}$ and they thus present the most promising platform to describe and control such effects. In these systems, the energies of CT states can be directly tuned through the use of different solvents. This concept has enabled the demonstration of fission

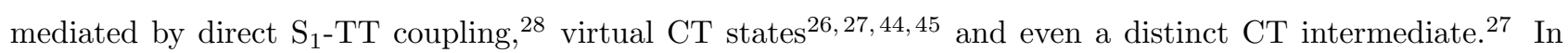
short, covalent dimers offer a versatile platform to systematically explore the $\mathrm{S}_{1}$ - $\mathrm{TT}$ transition and understand the nature of the coherent/incoherent pathways.

Here, we employ a combination of ultrafast spectroscopy and theoretical techniques to reveal the detailed mechanism of singlet fission in the orthogonal tetracene dimer DT-Mes (Figure 1 1 a) in solution and demonstrate how it is governed by a tuneable 'switch' between the incoherent and coherent regimes. Our experimental findings show that singlet fission in this system is a 'hot' process, i.e. it only occurs upon excitation distinctly above the band-edge of the first excited singlet state. ${ }^{46}$ Its mechanism is found to be qualitatively different depending on the solvent environment, and the fission efficiency is maximized in intermediate polarity solvents. Our theoretical analysis allows us to identify the crucial role of molecular vibrations to the singlet fission process, though alone they only give rise to weak mixing between states participating in singlet fission, and can only account for the experimental observations in low-polarity solvents where the process is incoherent. In more polar solvents we observe a qualitatively different regime of singlet fission, and we can only capture this behavior through the inclusion of dynamic solvent effects. Our model reveals that increasing the polarity switches DT-Mes into a regime of coherent singlet fission. However, contrary to previous studies reporting on superpositions of $\mathrm{S}_{1}$ and TT that dephase into the triplet pair, 6$] 7 \sqrt[9]{18}$ here we show that the coherent mechanism comes into play through the mixing of a CT state and TT. Coherent singlet fission occurs in solvents of intermediate polarity, where dynamic solvent effects induce the closest energetic proximity between these states so that molecular vibrations can mix them most efficiently. It is precisely the properties of CT states which allow us to tune them through the dielectric environment, which in contrast to local-excitations and triplet pairs, have a finite electric dipole. This underlines the novelty of our 'switch' mechanism, and emphasizes the potential of using CT states to tune coherent dynamics.

\section{Results and discussion}

Molecular structure and coupling. DT-Mes (chemical structure in Figure 1 1 ) consists of two tetracenes directly linked at the 5,5' position, with mesityl side groups for solubility. The synthesis and purification are described in SI section S2.1. Similarly to the anthracene ${ }^{47}$ and pentacene ${ }^{27}$ analogs and a closely related cyanosubstituted tetracene dimer ${ }^{48}$ DFT geometry optimization reveals that steric repulsion forces the two tetracenes into an orthogonal geometry. Being an alternate hydrocarbon, DT-Mes has no permanent dipole in the ground 
a

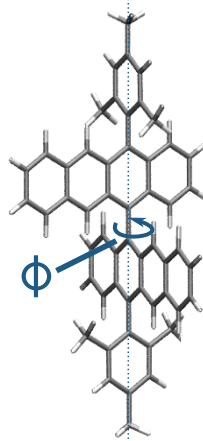

b
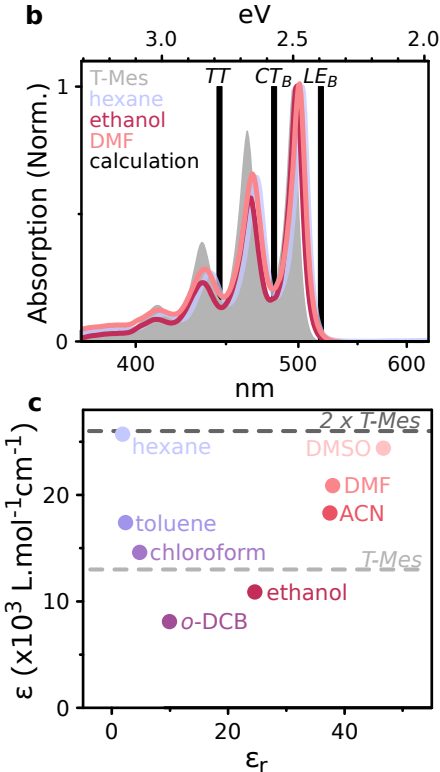

d

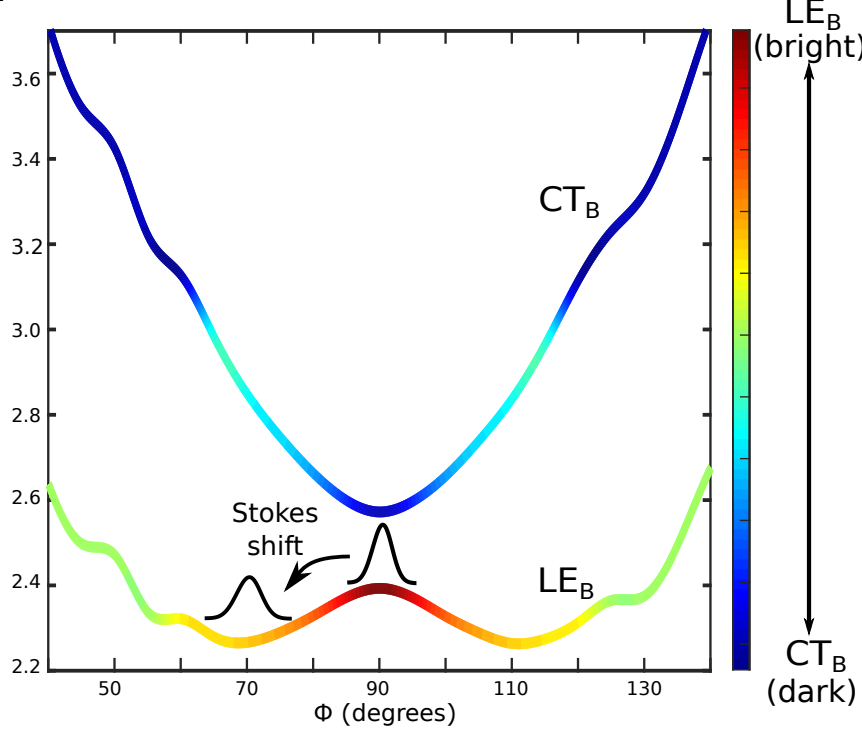

Figure 1 DT-Mes structure and photophysics. (a) Chemical structure of DT-Mes in its orthogonal ground-state geometry, indicating the dominant torsional motion. (b) Ground state absorption spectra of DT-Mes in solvents of different polarity and mesityl-substituted tetracene monomer T-Mes in chloroform (shaded). Calculated electronic energy levels are shown as vertical bars. The energy of the bright state $\mathrm{LE}_{B}$ is in excellent agreement with the position of the absorption onset. (c) Peak molar extinction coefficient of DT-Mes in different solvents compared to that of T-Mes in chloroform (dashed). The extinction coefficient is minimized in intermediate polarity solvents. (d) Potential energy surfaces of the two bright excited states along the torsional coordinate. The color code indicates the mixing between the $\mathrm{LE}_{B}$ and $\mathrm{CT}_{B}$ surfaces, leading to finite oscillator strength for the latter through intensity borrowing. The maximal mixing is found at the local minima of $\mathrm{LE}_{B}$, from where strongly red-shifted emission occurs.

electronic state, as predicted from the Coulson-Rushbrooke theorem. ${ }^{49}$ Therefore, the ground state geometry does not depend on the dielectric environment.

The orthogonal geometry raises the question of whether the two tetracenes can interact electronically, given their negligible $\pi$ overlap $27[44$ We determine the presence and relative strength of interchromophore coupling from the steady-state absorption (Figure 1p). This does not directly map onto the excited-state couplings between dark states (CT and TT) actually relevant to singlet fission, $\frac{38}{3}$ but it serves as a useful proxy! 10 Compared to the equivalent mesityl-tetracene monomer (shaded), the dimer (lines) exhibits a more prominent 0-0 absorption peak, though the exact 0-0/0-1 ratio varies with solvent. The enhanced 0-0 peak reflects J-type excitonic coupling between the short-axis-polarized $\mathrm{S}_{\mathrm{o}} \rightarrow \mathrm{S}_{1}$ transitions of the tetracenes. Moreover, the solvent dielectric constant has a powerful effect on the molar extinction coefficient (Figure 1k). In the extremes of our solvent polarity series we find that the dimer absorbs two times as strongly as the monomer. This would be consistent with negligible inter-tetracene interactions, similar to several prior reports of weakly coupled dimers $\sqrt[34]{37}$ However, in intermediate solvents the extinction coefficient of the dimer is less than that of even a single monomer, reaching as low as $40 \%$ of the expected value. This strong hypochromism is compelling evidence for strong interchromophore coupling, presumably mediated by interaction with dark charge-transfer (CT) states ${ }^{27}$ given the strong solvent dependence. This is further supported by the model approach which is developed in this work (see below), which reproduces the behavior of the molar extinction coefficient in different solvents due to mixing with the CT states (SI Section S1.3). As in the equivalent pentacene dimers $\stackrel{27}{44}$ we 
propose that the tetracenes are simultaneously coupled through CT and excitonic interactions. These have opposite effects on the energy of the bright state (a red-shift from the excitonic coupling and a blue-shift from the CT-mediated coupling), and the combination results in 'null aggregates' with weak spectral shifts and small changes in vibronic structure despite the significant interactions. $[50]$ Within this framework, the results in Figure 1 1 demonstrate that CT states play a central and tuneable role in the dimer electronic structure.

Exciton states and state mixing. In describing the electronic structure and properties of DT-Mes, we use as our basis the five lowest-energy adiabatic excited states in the orthogonal ground-state geometry, calculated in the absence of solvent effects. For a detailed definition of the electronic basis and full computational details please refer to SI Section S1.1. The molecule has approximate $C_{2}$ symmetry, thus the excited singlets transform either as the totally symmetric irreducible representation $A$ or the antisymmetric $B$. The two lowest-energy adiabatic singlets are the localized excitations $\mathrm{LE}_{B}$ and $\mathrm{LE}_{A}$, while the next two are the close-to-degenerate charge-transfer $\mathrm{CT}_{A}$ and $\mathrm{CT}_{B}$ states. The LE states refer to intra-monomer transitions, while CT states include

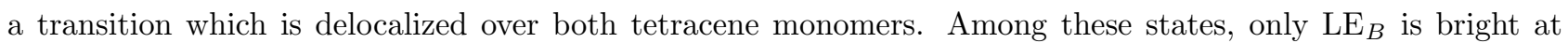
the Franck-Condon (FC) point. Its calculated energy (vertical bar in Figure 1b) is in excellent agreement with our experimental measurements. Likewise, the predicted energies of $\mathrm{CT}_{B}$ agree to within $\approx 0.1 \mathrm{eV}$ with the experimentally determined vacuum CT level. Apart from these single excitations, we approximate the triplet pair state resulting from singlet fission as the ground state quintet ${ }^{5} \mathrm{TT}$ (SI Section S1.1) of DT-Mes.

The above states constitute our electronic basis, however once semi-classical vibrational and/or solvent effects are taken into account (see below), a typical adiabatic excited state $|\Psi\rangle$, will, in general, become a superposition of these basis states:

$$
\begin{array}{r}
|\Psi\rangle=c_{\mathrm{LE}_{B}}\left|\mathrm{LE}_{B}\right\rangle+c_{\mathrm{LE}_{A}}\left|\mathrm{LE}_{A}\right\rangle+c_{\mathrm{CT}_{A}}\left|\mathrm{CT}_{A}\right\rangle+ \\
+c_{\mathrm{CT}_{B}}\left|\mathrm{CT}_{B}\right\rangle+c_{\mathrm{TT}}|\mathrm{TT}\rangle
\end{array}
$$

To make it easier to compare spectroscopic and theoretical results, we label these superposition states according to their dominant contribution, e.g. if $\left|\mathrm{LE}_{B}\right\rangle$ dominates the sum of Equation 11, we call the state $\mathrm{LE}_{B}$ for simplicity. We stress that this convention is not meant to imply that the state $|\Psi\rangle$ is exactly equal to one of the basis states, which are only present in their pure form in the ground-state geometry at low polarity. For such a superposition state $|\Psi\rangle$, the mixing between two of its basis states, e.g. $\mathrm{CT}_{B}$ and TT, is defined as:

$$
\rho_{\mathrm{CT}_{B} \mathrm{TT}}=c_{\mathrm{CT}}^{*} \cdot c_{\mathrm{TT}}
$$

It is obvious that this mixing will strongly depend on the choice of electronic basis, and it is always possible to work in a basis where it vanishes. Here we work in the basis of the diabatic states shown in Equation 1 , which are themselves equal to the adiabatic states at the FC point and in a non-polar solvent. Therefore, initial photoexcitation forms a pure $\mathrm{LE}_{B}$ state, and any subsequent mixing occurs between states that were originally separate, and is not an artifact of our choice of basis. 

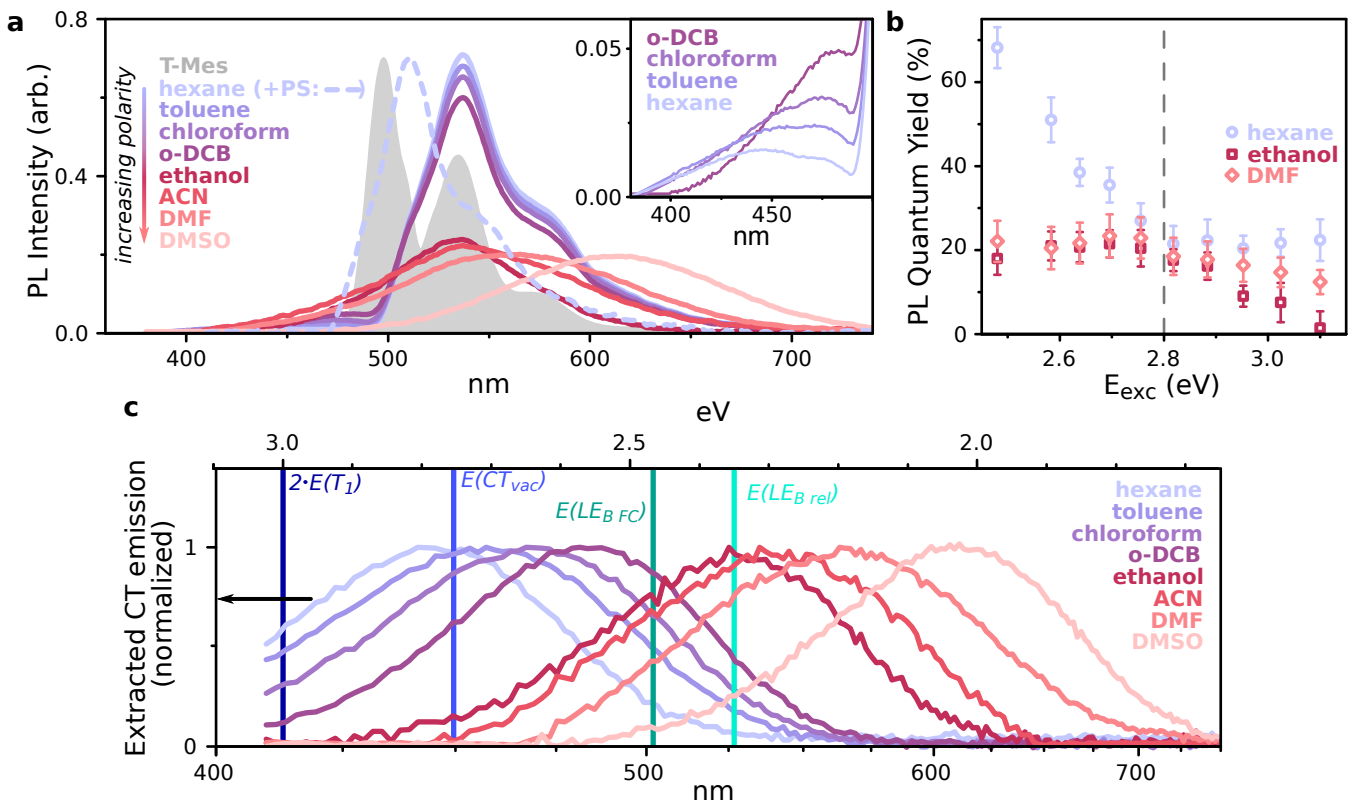

Figure 2 Multiple emissive species (a) Steady-state photoluminescence of DT-Mes in a range of solvents (solid lines), following excitation at $3.1 \mathrm{eV}$. The red-shift relative to monomer T-Mes (grey shaded) can be suppressed in high-viscosity solution (dashed). Inset: non-Kasha emission observed in non-polar solvents. (b) Solvent- and excitation-dependent photoluminescence quantum yield. In all media, the behavior of the system changes markedly for excitation at energies above 2.8 eV. (c) Energy landscape constructed from emission measurements. CT $\downarrow$ spectra (lines) are extracted from time-resolved emission measurements in each solvent (SI Figs. S27, S28). Vertical bars denote the energies of other relevant electronic states. LE ${ }_{B F C}$ and LE ${ }_{B \text { rel }}$ are determined in non-polar solvents with and without polystyrene. $E\left(\mathrm{~T}_{1}\right)$ is determined from sensitized phosphorescence (SI Fig. S9) and $E\left(\mathrm{CT}_{\text {vac }}\right)$ is extrapolated from the CT solvent dependence (see Figure 5 below). The energies of all emissive states are below the presumed singlet fission threshold $2 \cdot E\left(\mathrm{~T}_{1}\right)$.

To investigate the effect of molecular vibrations on the photophysics, we perform electronic structure calculations for a range of displacements along the torsional angle $\Phi$ between the monomers (Figure 1 1 a). This motion crucially underpins the photophysics of both the anthracene 47 and pentacene ${ }^{27}$ analogs. In DT-Mes, this is a low-frequency motion $\left(39.6 \mathrm{~cm}^{-1}\right)$ of $A$ symmetry. From analyzing the excitation character of the surfaces, we conclude that displacement along $\Phi$ leads to mixing between bright $\mathrm{LE}_{B}$ and dark $\mathrm{CT}_{B}$ (Figure 1 $\mathrm{d}$ ). This results in $\mathrm{CT}_{B}$ borrowing intensity from $\mathrm{LE}_{B}$ and becoming partially bright 51 Our calculations also reveal energetic relaxation of $\mathrm{LE}_{B}$ with increasing $\Phi$ and away from the $\mathrm{FC}$ point, to a minimum at $70^{\circ}$. This tendency towards planarization gives rise to Stokes-shifted emission (see below) and is also documented in the equivalent anthracene ${ }^{47}$ and pentacene ${ }^{27}$ dimers.

In a final clarification about notation, we point out that solvent effects result in mixing between the two symmetry-pure CT states. This results in the formation of two new CT states which are linear combinations of the originals. One of these has lower energy, which we denote as $\mathrm{CT}_{\downarrow}$, while the other one is destabilized and denoted as $\mathrm{CT}_{\uparrow}$. Both of these have finite $\mathrm{CT}_{B}$ character, hence both borrow intensity from $\mathrm{LE}_{B}$ and are partially bright, with the potential to emit photons. However, in practice we only detect photon emission from $\mathrm{CT}_{\downarrow}$. For ease we use this as the primary label for CT-related experimental signatures, with the recognition that it denotes a solvent-dependent mixture of $\mathrm{CT}_{B}$ and $\mathrm{CT}_{A}$. We discuss the nature and implications of this mixing in greater detail below, following the presentation of experimental data.

Multiple emissive species. We define the energetic landscape of DT-Mes through photoluminescence 
spectroscopy. Though the steady-state absorption spectra of monomer and dimer are almost identical (Figure 13), the steady-state photoluminescence of DT-Mes in solution (Figure 2a, solid lines) exhibits a large Stokes shift of $\approx 35 \mathrm{~nm}$ not observed in the monomer (shaded spectrum, $\approx 2 \mathrm{~nm}$ ). This shift can be reduced in high-viscosity polystyrene solution (dashed spectrum), confirming that it is linked to large-scale conformational change such as relaxation along $\Phi$. These measurements also show a more pronounced solvent dependence than the steady-state absorption. In the four least-polar solvents the emission is dominated by a well-defined vibronic progression $(500 \mathrm{~nm}-600 \mathrm{~nm})$ which does not shift and can be assigned to the relaxed (i.e. partially planarized) singlet $\mathrm{LE}_{B \mathrm{rel}}$. In the more polar solvents, the emission becomes featureless and progressively red-shifts with increasing polarity. This behavior is a hallmark of CT state emission 27/52 Similar features are also detected at the high-energy edge in non-polar solvents (inset), i.e. at shorter wavelengths than $\mathrm{LE}_{B}$ photoluminescence. This non-Kasha emission, from a state which is not the lowest-energy singlet in the molecule, is surprising, though long-lived high-energy CT states are known in similar orthogonal systems $\frac{53}{5}$ Here, it indicates the presence of multiple emissive species $\left(\mathrm{LE}_{B}\right.$ and $\left.\mathrm{CT}_{\downarrow}\right)$ in DT-Mes in non-polar solvents.

In non-polar solvents, it is possible to tune the balance of these species through the pump photon energy (full characterization in SI Section S2.9). Band-edge excitation in hexane yields purely excitonic emission, with a quantum efficiency of $\approx 68 \%$ (Figure $2 \mathrm{~b}$ ). With increasing photon energy, we detect a greater proportion of non-Kasha $\mathrm{CT}_{\downarrow}$ emission, as shown in the photoluminescence excitation maps in SI Figure S28. This changing balance is accompanied by a corresponding decrease in the photoluminescence quantum yield until saturation at $\approx 20 \%$. This is comparable to the quantum yield when the emission is dominated by $\mathrm{CT}_{\downarrow}$, as observed in all polar solvents following band-edge excitation. Interestingly, the behavior of the emission quantum efficiency qualitatively changes for excitation above the pump photon energy threshold of $2.8 \mathrm{eV}$ both for polar and nonpolar solvents - monotonic decrease and saturation, respectively. This behavior suggests a significant change in photophysical processes above this energy.

To guide our analysis of these excitation-dependent processes, we first compile the essential results from photoluminescence measurements to describe the DT-Mes energetic structure. Time-correlated single-photon counting (SI Section S2.9) reveals that in non-polar solvents the non-Kasha $\mathrm{CT}_{\downarrow}$ emission has a lifetime of $\approx$ 20 ns. This is comparable to what we observe in polar solvents, where $\mathrm{CT}_{\downarrow}$ is the only emissive species. This is significantly longer than the excitonic $\mathrm{LE}_{B}$ emission observed in polar solvents, which has a lifetime of $\approx$ $6 \mathrm{~ns}$. This large difference allows straightforward spectral decomposition to isolate the $\mathrm{CT}_{\downarrow}$ emission spectra, plotted for all solvents in Figure 2c. We compare this emission to the energies of key electronic states (vertical bars). Unsurprisingly, examination of the $\mathrm{CT}_{\downarrow}$ and excitonic energies reveals that $\mathrm{CT}$ emission dominates in the four polar solvents in which $\mathrm{CT}_{\downarrow}$ is the lowest-energy state. Comparing to Figure 1 ; , we also find that the minimum in extinction coefficient coincides with the point at which $E\left(\mathrm{CT}_{\downarrow}\right)$ is closest to $E\left(\mathrm{LE}_{B \mathrm{FC}}\right)$. This energetic proximity presumably enables the strongest CT-mediated coupling between tetracenes. Interestingly, sensitized phosphorescence measurements show that the dimerization motif of DT-Mes substantially destabilizes the triplet state from $1.3 \mathrm{eV}$ to $1.5 \mathrm{eV}$ (SI Figure S9), which is denoted by the vertical bar at $3 \mathrm{eV}$. This is a surprising effect which was also observed in orthogonal pentacene dimers, ${ }^{27}$ and its origin is not currently 

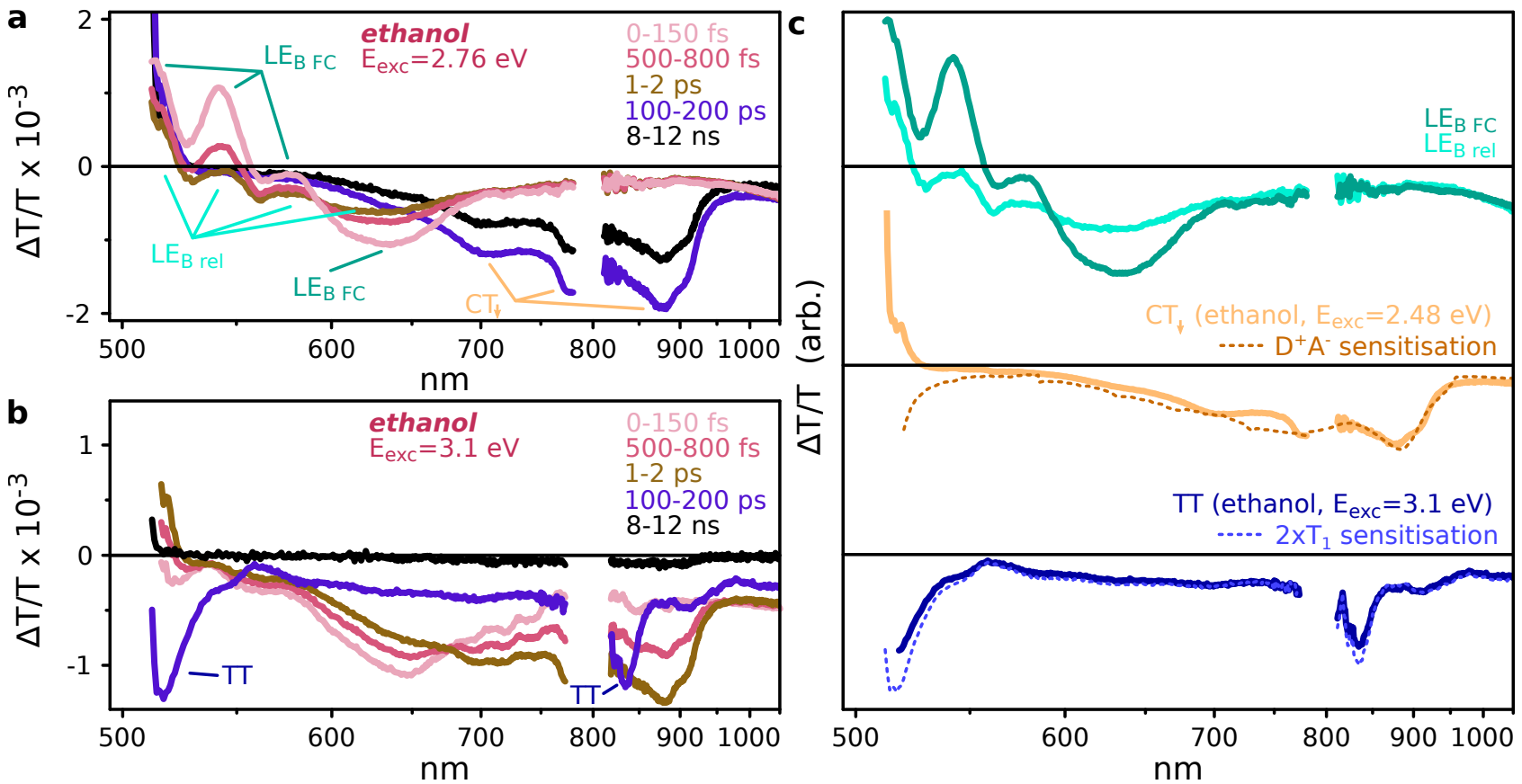

Figure 3 Excited-state evolution in DT-Mes. (a) Transient absorption spectra of DT-Mes in ethanol for excitation below the $2.8 \mathrm{eV}$ threshold. Signatures of $\mathrm{LE}_{B \mathrm{FC}}, \mathrm{LE}_{B \mathrm{rel}}$ and $\mathrm{CT}_{\downarrow}$ are indicated. (b) $E_{\text {exc }}>2.8 \mathrm{eV}$ generates a different initial state from lower-energy excitation. This state evolves into the same $\mathrm{CT}_{\downarrow}$ signatures (1 ps-2 ps) observed in a. Subsequent $\mathrm{CT}_{\downarrow}$ decay yields distinctive TT signatures (indicated), which largely decay $<1 \mathrm{~ns}$. (c) Species-associated spectra extracted from transient absorption spectroscopy (solid) and reference spectra from charge and triplet sensitization (dashed). The relative magnitudes of the spectra reflect their relative molar extinction coefficients. See SI Section 2.11 for details.

understood. We recall, though, that previous studies on perylene diimide films found the triplet energy varies with intermolecular coupling,, 54 and significant energy shifts have been reported between TIPS-tetracene ${ }^{55}$ and a phenyl-substituted derivative. 56 There is evidently significant scope - currently little explored - to tune the triplet energy on the same parent chromophore. The crucial result of these measurements for our purposes here is that none of the states observed in absorption or emission approach the energy expected to be required for singlet fission. Nor is it immediately evident what is the origin of the observed thresholding behavior appearing at $2.8 \mathrm{eV}$ in Figure $2 \mathrm{~b}$, since it would not be expected to arise from singlet fission.

CT-mediated singlet fission. To better understand the system's behavior and the role of dark electronic states, we have probed the dynamics of DT-Mes using transient absorption spectroscopy in all eight solvents with ten pump photon energies, spanning from band-edge excitation $(2.48 \mathrm{eV})$ to significant excess energy $(3.10 \mathrm{eV})$. We present representative results for ethanol solution in Figure 3, with detailed spectra and kinetics available in SI Section S2.6. Below the $2.8 \mathrm{eV}$ excitation threshold (Figure $3 \mathrm{a}$ ) we can identify the signatures of three distinct electronic states. The initial state $\mathrm{LE}_{B \mathrm{FC}}$ is characterized by a prominent trio of stimulated emission $(\Delta T / T>0)$ peaks at $510 \mathrm{~nm}, 540 \mathrm{~nm}$ and $580 \mathrm{~nm}$ and a well-defined excited-state absorption $(\Delta T / T<0)$ at $630 \mathrm{~nm}$. These signatures evolve with $\approx 550 \mathrm{fs}$ time-constant to a state with strongly attenuated stimulated emission and a broader, flattened excited-state absorption. In corresponding transient grating photoluminescence ${ }^{57}$ measurements (SI Section S2.8), we observe on precisely the same timescale a pronounced redshift of the emission consistent with geometric relaxation of the excited state. This $550 \mathrm{fs}$ time constant is intermediate between the 
planarization dynamics reported for the equivalent anthracene $e^{58}$ and pentacene $e^{27}$ dimers. We can thus assign the second species in transient absorption to the partially planarized singlet $\mathrm{LE}_{B \text { rel }}$. On longer timescales a new species is evident with unique excited-state absorption in the near-infrared (e.g. $710 \mathrm{~nm}, 775 \mathrm{~nm}, 880 \mathrm{~nm}$ ). This state is similarly long-lived to the $\mathrm{CT}_{\downarrow}$ emission $(\approx 20 \mathrm{~ns})$ and the features in the near-infrared closely match peaks observed in the chemical oxidation and reduction spectra (Figure 3e and SI Section S2.11), allowing assignment to the CT state. While it contains multiple radical anion and cation signature peaks, the imperfect match in Figure 3e also shows that $\mathrm{CT}$ is not a pure $\mathrm{D}^{+} \mathrm{A}^{-}$state. We attribute the deviations from the $\mathrm{D}^{+} \mathrm{A}^{-}$ spectrum to LE contributions to the total wavefunction, which also give the state the ability to emit. In short, we observe a simple excited-state progression from the initial bright singlet state $\mathrm{LE}_{B \mathrm{FC}}$ to a conformationally relaxed singlet $\mathrm{LE}_{B \mathrm{rel}}$, and from there to a long-lived emissive CT state. Similar dynamics are observed in all solvents, for excitation below $2.8 \mathrm{eV}$ but above the energy of the $\mathrm{CT}_{\downarrow}$ state (which varies by solvent). We do not detect any signatures of triplet excitons (Figure 3 , bottom) in these conditions, demonstrating that singlet fission is inactive and intersystem crossing from LE and CT states is inefficient.

For excitation above the $2.8 \mathrm{eV}$ threshold, Figure $3 \mathrm{~b}$, the initial excited-state absorption signature is distorted from that of $\mathrm{LE}_{B \mathrm{FC}}$, exhibiting much weaker stimulated emission. Nonetheless, it rapidly evolves to the same CT state identified in Figure 3a. The latter now undergoes a new decay pathway. At 100 ps-200 ps we detect the unique excited-state absorption fingerprint of DT-Mes triplets at $520 \mathrm{~nm}$ and $830 \mathrm{~nm}$, identified from solution sensitization (Figure $3 \mathrm{c}$ and SI Section S2.10). The lifetime of these triplets is $\approx 800 \mathrm{ps}, 5000$ times shorter than the lifetime of individual triplets in sensitization (SI Fig. S26). We are only able to explain such a significant reduction in the triplet lifetime through triplet-triplet annihilation. At the low concentrations and excitation densities used in our experiments, that requires two triplet excitons to be produced on a single DT-Mes molecule, ruling out intersystem crossing from LE states 59 or triplet generation from CT recombination $\sqrt{60}$ as possible formation mechanisms. Instead, we consider this rapid annihilation of triplet pairs to be a hallmark of intramolecular singlet fission, as previously observed in numerous dimer and conjugated-polymer

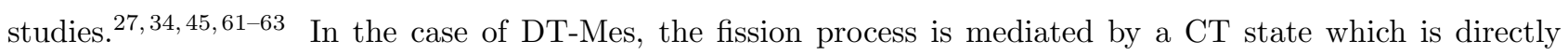
populated and presents distinct spectroscopic features. Though often predicted, 1,31 this fission mechanism has proven experimentally elusive and has only been conclusively identified in one other system, a similar orthogonal pentacene dimer with TIPS solubilizing groups. ${ }^{27}$ It is far more common for CT states to modulate singlet fission through a 'virtual' or superexchange pathway,, $26 \sqrt[27]{29} \mid \frac{44}{45}$ but the directly observable CT intermediate here allows us to obtain deeper mechanistic insight.

Singlet fission yield variation. We find that the basis spectra identified in Figure 3 are sufficient to describe the excited-state progression in all solvents and at all pump photon energies, enabling easy comparison between experimental conditions. We highlight in Figure 4 how the terminal state branches between fissiongenerated TT and long-lived CT at 100 ps-200 ps. At this time delay singlet fission - when it occurs - is complete. In panel a we observe that the TT yield is clearly optimized for intermediate solvents such as ethanol and $o$-DCB. These are the solvents where the CT and $\mathrm{LE}_{B}$ energies most closely approach (Figure 2 c) and where we infer the strongest LE-CT coupling from the low oscillator strength (Figure 1 


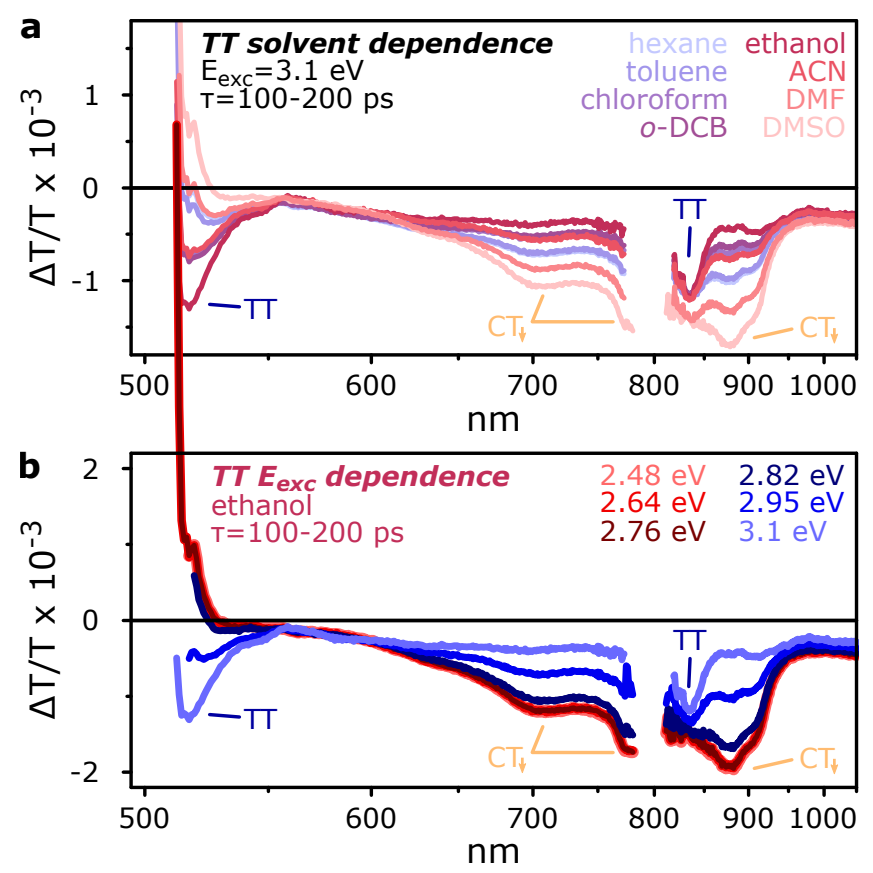

Figure 4 | Tuning the TT-CT balance. (a) Solvent dependence of TT yield at $100 \mathrm{ps}-200 \mathrm{ps,} \mathrm{following} \mathrm{excitation} \mathrm{at} 3.1 \mathrm{eV}$. The TT yield changes depending on the dielectric environment, and is maximized in intermediate polarity solvents. (b) Pump photon energy dependence of TT yield at $100 \mathrm{ps}-200 \mathrm{ps}$ in ethanol. TT forms for excitation above $2.8 \mathrm{eV}$, and its yield increases with excitation energy up to $3.1 \mathrm{eV}$. The primary features of TT and $\mathrm{CT}_{\downarrow}$ are indicated again for clarity.

lower polarity, we detect increased branching into the long-lived $\mathrm{CT}_{\downarrow}$ state. Moreover, in ethanol the branching into TT increases monotonically with pump photon energy up to a maximum of $3.1 \mathrm{eV}$ (panel b, instrument limit). The TT yield is thus a complex function of both excitation energy and solvent. The optically activated singlet fission reported in Figure $4 \mathrm{~b}$ is particularly unusual. It has never before been observed in covalent dimers, and while it recalls the behavior of certain conjugated polymers $\underline{46|63| 64}$ the important distinction is that the activated process is not fast. Indeed, in some of the solvents singlet fission proceeds over tens of ps, a remarkably long timescale for 'hot' dynamics, which we discuss further below.

Using the excited-state signatures identified in Figure 3;, we can determine the yields of TT and long-lived $\mathrm{CT}$ obtained in all of our transient absorption datasets. These are presented in the action spectra in Figure $5 \mathrm{~b}$, which summarize the full solvent- and excitation-dependent behaviors highlighted in Figure 4. The orangeshaded spectra reproduce the $\mathrm{CT}$ emission from Figure 22. In polar solvents where $E\left(\mathrm{CT}_{\downarrow}\right)<E\left(\mathrm{LE}_{B \mathrm{FC}}\right)$ (right), the $\mathrm{CT}$ action spectra (circles) reveal that initial conversion into $\mathrm{CT}$ is always quantitative. In the nonpolar solvents (left), the threshold for $\mathrm{CT}$ formation roughly follows the envelope of $\mathrm{CT}_{\downarrow}$ emission, suggesting that this emission lineshape is a reasonable proxy for the electronic energy of the state. This result demonstrates that if the initial excitation has greater energy than some portion of the broader $\mathrm{CT}_{\downarrow}$ distribution, then the system will access that state. We note that in non-polar solvents the total yields do not sum to $100 \%$. In these solvents the initial formation of $\mathrm{CT}_{\downarrow}$ competes with relaxation into $\mathrm{LE}_{B \text { rel }}$, which we can detect in transient absorption (SI Figure S36) and the vibronically structured emission (Figure 2a). This state exhibits a lifetime of $\approx 6$ ns and evidently does not undergo singlet fission (SI Figure S30). The yield of LE $_{B \text { rel }}$ is equivalent to the difference between the presented yields and $100 \%$. 


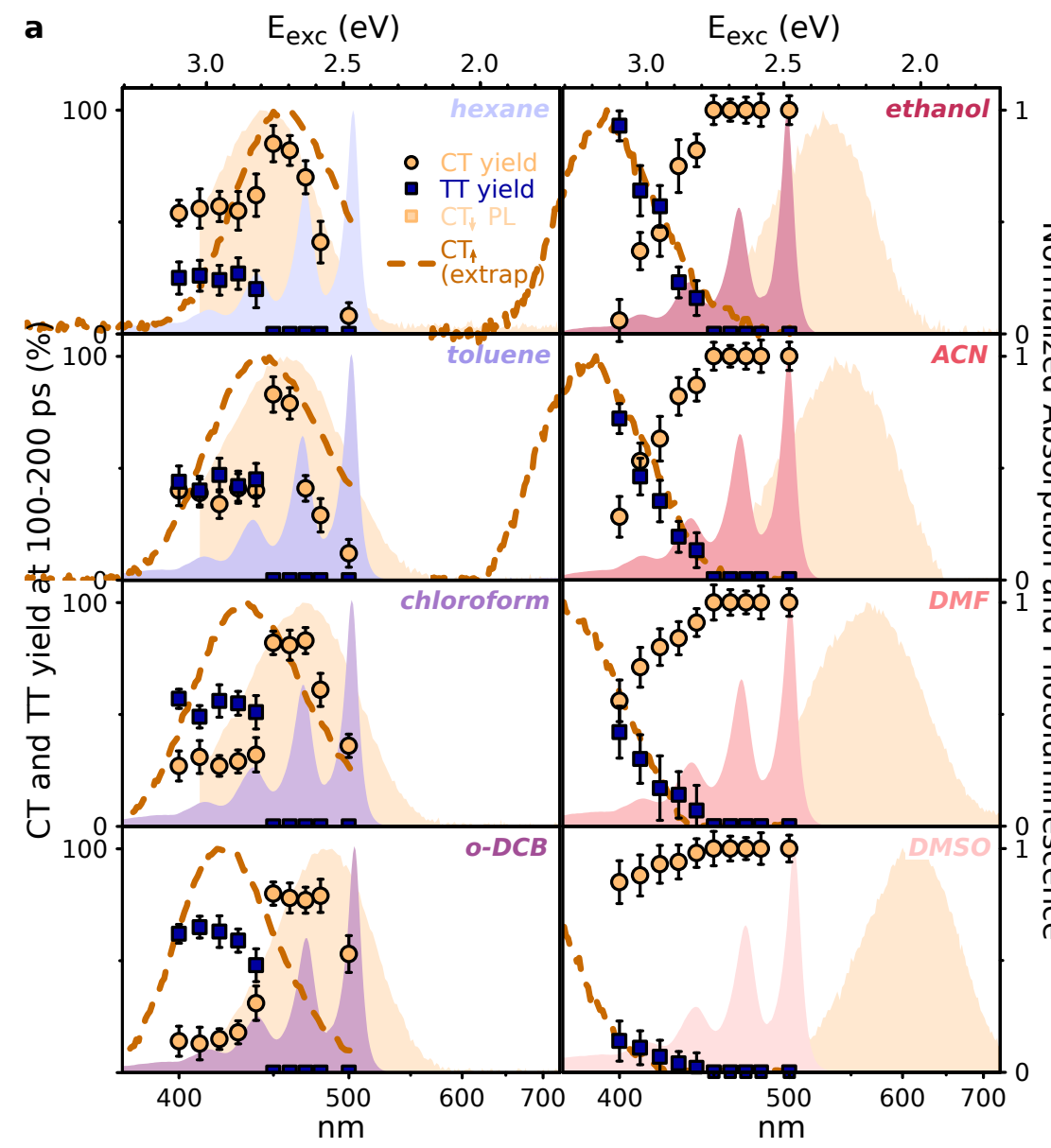

b

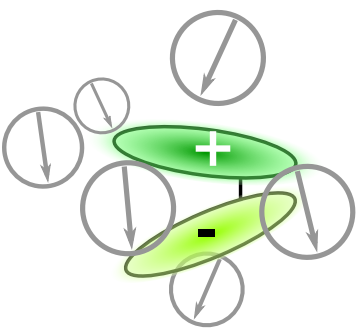

$\mathbf{C T}_{\uparrow}$ : destabilizing solvent dipole coupling
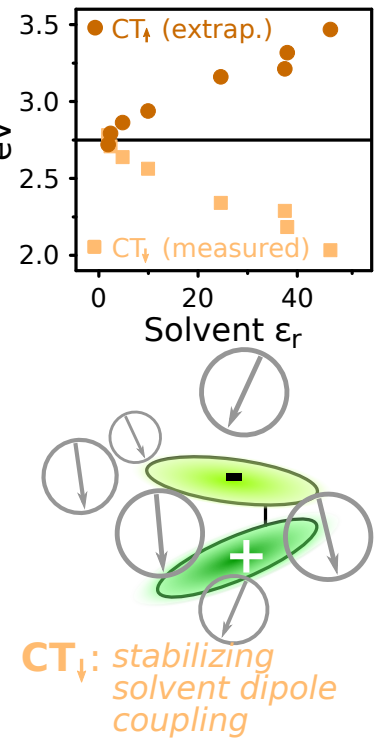

Figure 5 | TT and CT action spectra. (a) Yield of long-lived $\mathrm{CT}_{\downarrow}$ (circles) and TT pairs (squares), extracted from excitationdependent transient absorption measurements at $100 \mathrm{ps}-200 \mathrm{ps}$ time delay, for solvents of increasing polarity (left to right). The $\mathrm{TT}$ cross-section was determined by triplet sensitization, assuming $\mathrm{TT} \approx 2 \cdot \mathrm{T}_{1}$. The $\mathrm{CT}_{\downarrow}$ yield is relative to the yield in ethanol following band-edge excitation, which we assume to be $100 \%$. See SI Section S2.11 for details. Shaded spectra are steady-state absorption and extracted $\mathrm{CT}_{\downarrow}$ emission from Figure 2. Dashed spectra approximate the $\mathrm{CT}_{\uparrow}$ distribution, by reflecting the $\mathrm{CT}_{\downarrow}$ emission spectrum across the vacuum CT energy $\mathrm{CT}_{\text {vac }}$ (see main text and panel b). (b) Peak of CT emission (squares) as a function of solvent $\epsilon_{r}$ allows extrapolation of the vacuum $\mathrm{CT}$ energy $\approx 2.75 \mathrm{eV}$ (blue). $\mathrm{CT}_{\downarrow}$ is stabilized from this level by interactions with the solvent shell (bottom), and the oppositely polarized state would be equally destabilized (top), giving rise to the $\mathrm{CT}_{\uparrow}$ state (circles).

In every solvent, regardless of the threshold energy for CT formation, we observe the same threshold energy for singlet fission of $\approx 2.8 \mathrm{eV}$. It is noteworthy that this energy is significantly lower than the expected fission threshold of $2 \cdot E\left(\mathrm{~T}_{1}\right) \approx 3.0 \mathrm{eV}$. It is common to invoke an entropic driving force to explain such endothermic fission in solid-state systems, $\frac{16][1][65}{6}$ but that should not be a factor in a strictly dimeric system. Instead, we can only rationalize this low onset energy through a binding energy. Our results imply that the immediate product of singlet fission is a bound triplet-pair state $\sqrt[16] 66] 68]{68}$ The spin-singlet triplet pair can be significantly stabilized relative to two 'free' triplets due to mixing of the diabatic TT wavefunction with other LE and CT

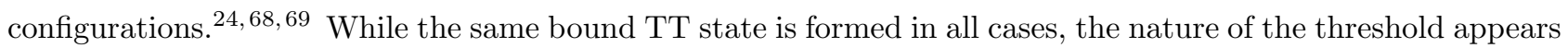
to change from non-polar (Figure 5 left) to polar (Figure 5 right) solvents. In non-polar solvents the TT yield is nearly constant above the threshold. In polar solvents, the TT yield shows a distinctly gradual onset. We can explain this phenomenon using the pair of $\mathrm{CT}$ states illustrated schematically in Figure $5 \mathrm{~b}$. Here we recall that 
the emissive CT state is stabilized by interactions with the solvation shell (bottom), hence it red-shifts as the solvent dielectric constant increases. From the solvent dependence of the CT emission we can then extrapolate the vacuum CT energy as $2.75 \mathrm{eV}$ : this corresponds to the bare electronic energy of the CT state. We propose that for a given solvation shell there exists an oppositely polarized CT state (top) which will be destabilized by the same degree, though that state is not necessarily populated. To approximate the energy distribution of this $\mathrm{CT}_{\uparrow}$ state, we simply reflect the $\mathrm{CT}$ emission spectrum about the $\mathrm{CT}_{\text {vac }}$ level. This process generates the $\mathrm{CT}_{\uparrow}$ spectrum we plot in dashed lines in Figure 5a. Surprisingly, this graphical approach provides a remarkably good fit to the activation data. Our results suggest that singlet fission in polar solvents is mediated specifically by a destabilized 'upper' CT state, which to our knowledge has never before been observed or even suggested. To understand this behavior and the possible role such a destabilized CT state could play, we return to our theoretical description of DT-Mes.

Modelling of vibrational and solvent effects. The experimental results of the previous sections show that vibrations must be considered to explain the excited state photophysics of DT-Mes: the first step observed in transient absorption measurements is relaxation along $\Phi$ (Figure 3), and suppressing this channel through the use of polystyrene has a significant effect on the emission properties (Figure 2 a). Furthermore, as summarized in Figure 5 , there is a qualitative difference in the singlet fission mechanism in non-polar versus intermediateand highly-polar solvents, while the final triplet yield also depends strongly on the excitation energy. These properties are all uncommon among singlet fission systems. It is therefore important to incorporate vibrational and solvent effects into our model description of DT-Mes, as well as the role of excess energy. We lay out here the principles of our approach; full details of the model Hamiltonian are given in SI Section S1.2.

The approximate $C_{2}$ symmetry of DT-Mes places important constraints on the fission mechanism. The TT state, which we calculate as the ground state quintet ${ }^{5}$ TT (SI Section S1.1), is $A$-symmetric. So is the $\Phi$ rotation of Figure 1 , meaning it only mixes states of the same symmetry. Therefore, neglecting two-electron contributions, 129 [1 31 TT only mixes with $\mathrm{CT}_{A}$ along this coordinate. However, only the bright $B$-symmetry states $\mathrm{LE}_{B}$ and $\mathrm{CT}_{B}$ are optically accessible. The transition from these states to TT must thus be accomplished through some form of symmetry breaking. Physically, such symmetry breaking could be provided by the vibronic coupling along a $B$-symmetric mode. We take $\eta=20 \mathrm{meV}$ as a representative maximum value for this vibronic coupling, similar to values obtained for inter-molecular symmetry-breaking modes in rubrene ${ }^{9}$ We introduce $\eta$ in our model as the coupling of $A$ - and $B$-symmetry states to each other. Therefore, a model Hamiltonian which includes the effect of the torsion and symmetry-breaking mode on the electronic states, in the basis of $\left\{\mathrm{LE}_{B}, \mathrm{LE}_{A}, \mathrm{CT}_{B}, \mathrm{CT}_{A}, \mathrm{TT}\right\}$ is:

$$
H_{e l}=\left[\begin{array}{ccccc}
E_{\mathrm{LE}_{B}}(\phi) & \eta & J(\phi) & 0 & 0 \\
\eta & E_{\mathrm{LE}_{A}}(\phi) & 0 & 0 & 0 \\
J(\phi) & 0 & E_{\mathrm{CT}_{B}}(\phi) & \eta & 0 \\
0 & 0 & \eta & E_{\mathrm{CT}_{A}}(\phi) & \Lambda(\phi) \\
0 & 0 & 0 & \Lambda(\phi) & E_{\mathrm{TT}}(\phi)
\end{array}\right] .
$$



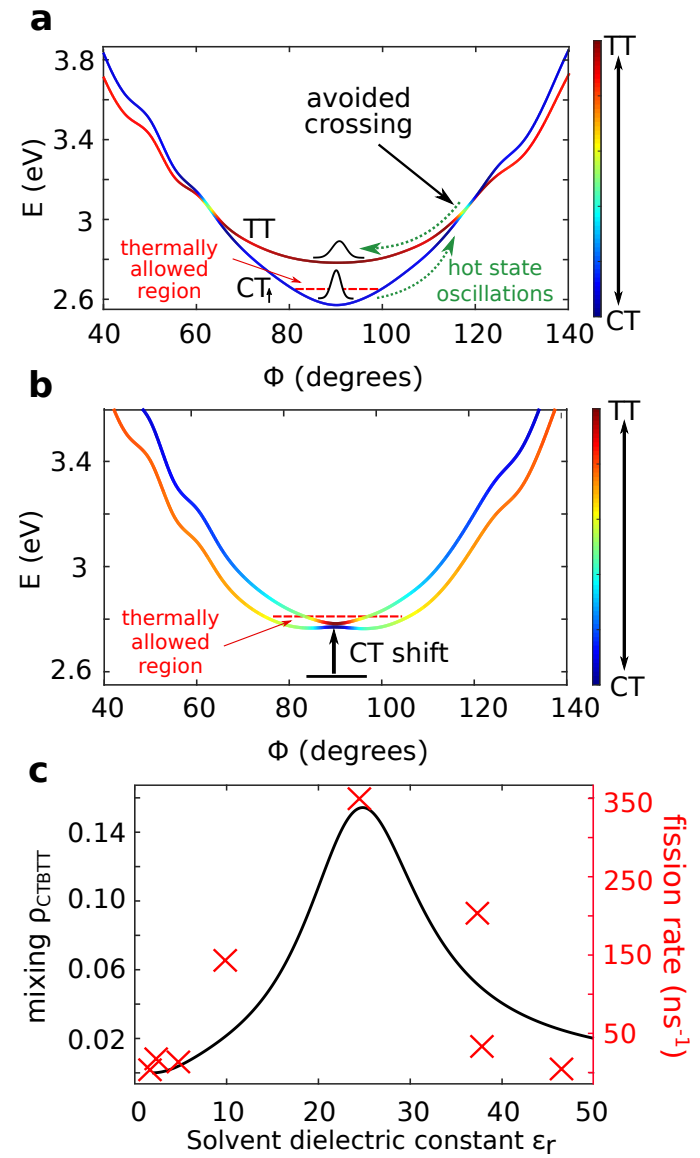

Figure 6 Coherent and incoherent singlet fission. (a) Potential energy surfaces in non-polar solvents (e.g. hexane). CT $\uparrow$ does not mix with TT (color scale) in the thermally allowed region at room temperature, the boundaries of which are indicated with the red dashed line. Incoherent singlet fission takes place when enough excess energy to reach the avoided crossing to the $\mathrm{TT}$ surface is provided. (b) Potential energy surfaces for intermediate-polarity solvents (e.g. ethanol). CT $\uparrow$ is destabilized (arrow) and approaches TT, and the two are strongly mixed by the dihedral oscillation (color scale), resulting in coherent singlet fission. Exciting $\mathrm{CT}_{\uparrow}$ with more excess energy leads to superposition states with stronger TT contributions than within the thermally allowed region of $\mathrm{CT}_{\uparrow}$ (red dashed line). (c) Mixing between bright $\mathrm{CT}$ and TT as a function of solvent $\epsilon_{r}$, at the fixed angle of $\Phi=80^{\circ}$. Intermediate-polarity solvents result into maximal mixing and coherent singlet fission. This is compared with the experimental singlet fission rate, which we extract from the TT growth kinetics in SI Section S2.6. and the TT yields in Figure 5 The rate is optimized in solvents where singlet fission is coherent.

Here $J(\phi)$ and $\Lambda(\phi)$ are the couplings between states of the same symmetry along the symmetric torsional mode.

Due to the negligible Huang-Rhys factors of antisymmetric $B$ modes compared to symmetric ones, their Franck-Condon factors and displacements are orders of magnitude smaller than those of $A$ modes ${ }^{70-72}$ Therefore, within our model approximation, we consider excess energy to only result in the displacement of $A$ symmetry modes, and to not generate any additional $B$ symmetry vibrations. Since the singlet fission timescale in DT-Mes is relatively long ( $>10 \mathrm{ps}$ ), we expect high-frequency $A$ modes displaced via excess energy excitation to have mostly relaxed towards lower-frequency modes at these timescales. We further simplify the problem by approximating the resulting low-frequency mode distribution only in terms of motion along $\Phi$. Consequently, within this model approach, excess-energy excitation leads to larger-amplitude oscillations along $\Phi$, accessing higher regions of that surface. Importantly for this picture, the initial step of vibrational relaxation does not remove energy from the molecule but only redistributes it into other motions. These are damped through subsequent vibrational cooling, which for isolated molecules in solution is mediated by solvent-solute interactions, 
e.g. collisions between DT-Mes and solvent molecules. This diffusion-mediated cooling can require many tens of ps. ${ }^{73}$ 76 $T$ The excess vibrational energy in this 'hot' state manifested as large-amplitude $\Phi$ oscillations can thus persist on timescales relevant to singlet fission.

We now turn our attention to incorporating solvent effects into our description. In a polar solvent, local electric fields may be randomly oriented, leading to an energetic separation of the two CT states which inevitably appear in such a dimer molecule, with dipoles pointing in opposite directions. We refer to the stabilized and destabilized $\mathrm{CT}$ states as $\mathrm{CT}_{\downarrow}$ and $\mathrm{CT}_{\uparrow}$. These states are symmetry-broken, as the solvent can generally arrange itself in a non-symmetric fashion around DT-Mes. This phenomenon is mathematically captured by introducing an additional coupling $\Delta$ between symmetry-pure $\mathrm{CT}_{A}$ and $\mathrm{CT}_{B}$, which mixes them into the new eigenstates:

$$
H_{e l}=\left[\begin{array}{ccccc}
E_{\mathrm{LE}_{B}}(\phi) & \eta & J(\phi) & 0 & 0 \\
\eta & E_{\mathrm{LE}_{A}}(\phi) & 0 & 0 & 0 \\
J(\phi) & 0 & E_{\mathrm{CT}_{B}}(\phi) & \eta+\Delta & 0 \\
0 & 0 & \eta+\Delta & E_{\mathrm{CT}_{A}}(\phi) & \Lambda(\phi) \\
0 & 0 & 0 & \Lambda(\phi) & E_{\mathrm{TT}}(\phi)
\end{array}\right] .
$$

Physically, this expresses the fact that in the presence of an electric field it is the CT states with a permanent dipole and not the symmetry-adapted CT states which are eigenstates of the system.

We can determine the values of $\Delta$ corresponding to different solvent $\epsilon_{r}$ by benchmarking against the measured energetic stabilization $\mathrm{CT}_{\downarrow}$ (Figure 5b). For intermediate-polarity solvents, we find that $\mathrm{CT}_{\downarrow}$ and $\mathrm{LE}_{B}$ are energetically very close, leading to a strong mixing and transfer of oscillator strength from LE to CT, i.e. intensity borrowing! 77 This description closely reproduces the experimental trend of Figure 1 for the molar extinction coefficient, which is minimized in intermediate-polarity solvents. The calculated molar extinction coefficient is shown in SI Section S1.3 for a range of solvents. If the equivalently destabilized state $\mathrm{CT}_{\uparrow}$ were to form, we would in principle expect it to relax into $\mathrm{CT}_{\downarrow}$. However, this process should occur through reorganization of the solvent shell and would be expected to occur on the few- to tens-picosecond timescale. ${ }^{78}$ Accordingly, we would not expect to detect any photon emission from $\mathrm{CT}_{\uparrow}$, since the relevant emission lifetime is tens of nanoseconds (for $\mathrm{CT}_{\downarrow}$, SI Section S2.9). This timescale remains sufficiently long, though, for $\mathrm{CT}_{\uparrow}$ to play an important role in singlet fission despite being an unstable state.

Coherent and incoherent singlet fission. We can use this framework for the DT-Mes electronic structure to rationalize the surprising behavior in Figure 5. Our transient absorption experiments reveal TT is never formed directly from initial $\mathrm{LE}_{B}$ but is always preceded by CT states (Figure 3b and SI Section S2.6), and it only forms in conditions where $\mathrm{CT}$ is already formed with high efficiency (Figure 5 a). The only major distinction between fission regimes occurs in the subsequent transition, from CT to TT, where the symmetry of the system must be broken. This step is the chief focus of our analysis, and we consider two limiting cases. In low-polarity solvents, $\eta>\Delta$ and vibronic effects provide the symmetry-breaking needed to access TT. In intermediatepolarity solvents, $\eta<<\Delta$ and the symmetry-breaking is dominated by solvent effects. In either case, the CT states formed may initially be vibrationally 'hot' due to excess-energy excitation. Moreover, potentially either 
Figure 7 Adiabatic potential energy surfaces along the dihedral angle $\Phi$ and the solvent dielectric constant, showing their CT (blue) and TT (red) character. For low-polarity solvents the surfaces only mix for large-amplitude oscillations along $\Phi$ in the vicinity of an avoided crossing, leading to incoherent fission. Strong mixing is induced in intermediate-polarity solvents (e.g. ethanol), making singlet fission coherent. Finally,very polar solvents once again lead to reduced mixing between the surfaces.

$\mathrm{CT}_{\downarrow}$ or $\mathrm{CT}_{\uparrow}$ may be present, since both are mixed with $\mathrm{CT}_{B}$ and thus accessible from $\mathrm{LE}_{B}$. A Marcus-like analysis of the $\mathrm{CT}_{\uparrow}$ to $\mathrm{TT}$ transition is discussed in SI section S1.4, along with its limitations.

The $\mathrm{CT}_{\uparrow}$ and $\mathrm{TT}$ potential energy surfaces along $\Phi$ for low-polarity solvents are given in Figure 6 a, coded for their $\mathrm{CT} / \mathrm{TT}$ character. In this case, $\mathrm{CT}_{\uparrow}$ and $\mathrm{CT}_{\downarrow}$ are almost degenerate. $\mathrm{CT}_{\uparrow}$ does not develop any $\mathrm{TT}$ character within the region accessible at room temperature (nor does $\mathrm{CT}_{\downarrow}$ ), and the TT surface also remains pure. Within this model, a transition between the states is possible only through the avoided crossing that appears at larger angles, making singlet fission incoherent $9[18$ Excitation of a hot state which can access that part of the potential energy landscape corresponds to an excess energy of about $0.4 \mathrm{eV}$. Excitation with still greater energy would not have a significant effect on the triplet yield, as the mixing is always negligible away from the crossing and no additional $B$-type (i.e. coupling) vibrations are generated. This is in close agreement with the experimental observation that a sharp threshold for TT formation appears at $0.33 \mathrm{eV}$ of excess-energy excitation (Figure 5), with little dependence above that.

In intermediate-polarity solvents, the transient solvation dynamics which stabilize $\mathrm{CT}_{\downarrow}$ also destabilize $\mathrm{CT}_{\uparrow}$ to near the energy of TT. This results in a mixing of the two states through the dihedral rotation along $\Phi$, as shown in Figure 6b. The contribution of each component varies along the potential energy surface, with the TT character (red shading) increasing away from the FC point. This is the regime of coherent singlet fission, as defined in previous studies. ${ }^{[}$Hence exciting the system at higher energies leads to superposition states which are more 'TT rich', leading to stronger couplings to the final, relaxed TT state. A superposition of TT and $\mathrm{CT}_{\uparrow}$ eventually dephases towards its constituents, which are the relevant eigenstates of the system at its equilibrium 


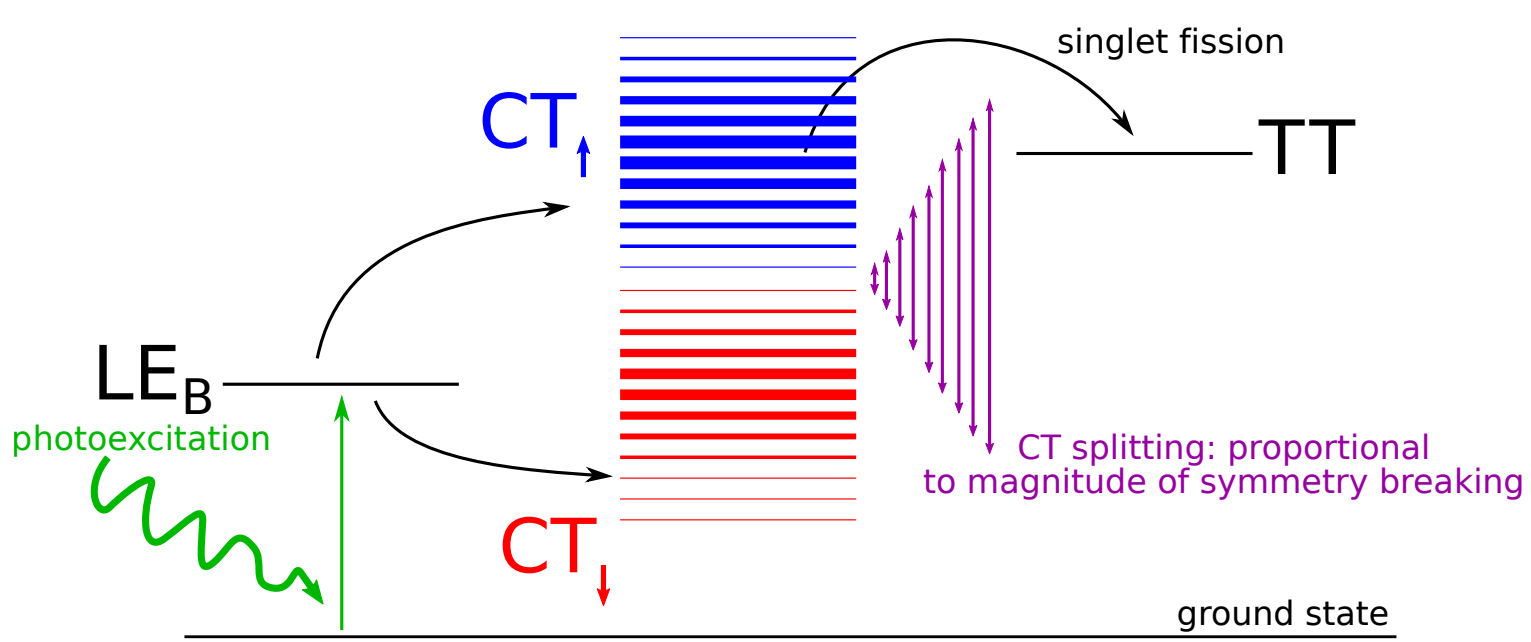

Figure 8 Switching the singlet fission mechanism. Following photoexcitation to the bright $\mathrm{LE}_{B}$ state, the $\mathrm{CT}_{\uparrow}$ and $\mathrm{CT}_{\downarrow}$ states get populated. The thickness of these two states denotes their mixing with TT and LE $\mathrm{T}_{B}$ respectively. Symmetry-breaking interactions (solvent effects in our study) control their splitting and consequently the mixing with the other electronic states. Coherent singlet fission takes place in the regime of high mixing between $\mathrm{CT}_{\uparrow}$ and TT.

geometry. The larger the TT contribution, the more likely it is that the system will collapse towards the final, relaxed $\mathrm{TT}$ state. In this regime the destabilized $\mathrm{CT}_{\uparrow}$ state is the 'gateway' for singlet fission regardless of excitation energy, and we would thus expect the TT yield to track the accessibility of $\mathrm{CT}_{\uparrow}$. Experimentally, this would translate into the energy distribution inferred in Figure 5 a (right), which is exactly what is observed. At the same time, regions of the surface with high TT contributions exhibit reduced CT character, leading to an anti-correlation of the TT and long-lived CT yields.

The increased degree of mixing between the CT and TT surfaces with increasing solvent polarity is reflected in the fission rates shown in Figure 6r, extracted from the TT growth kinetics in the transient absorption data in SI Section S2.6 and the TT yields in Figure 5a. Initially, increasing the solvent polarity increases the rate of singlet fission, but in very polar solvents the TT formation rate drops again to low values. In these solvents $\mathrm{CT}_{\uparrow}$ shifts above the $\mathrm{TT}$ surface and the two stop mixing, leading to a regime of incoherent fission similar to the non-polar case. This mixing is quantified via $\rho_{\mathrm{CT}_{B} \mathrm{TT}}$ of Equation 2, which we plot in Figure 6e for the fixed angle $\Phi=80^{\circ}$ as a function of solvent dielectric constant. Note that this is calculated in the original basis of symmetry-pure $\mathrm{CT}$ states which form $\mathrm{CT}_{\uparrow}$. In summary, we find that intermediate-polarity solvents lead to maximal mixing between $\mathrm{CT}_{B}$ and $\mathrm{TT}$, thus leading to coherent singlet fission. In this case, $\mathrm{TT}$ is formed via the dephasing of the $\mathrm{CT}_{\uparrow} / \mathrm{TT}$ superposition, which is more efficient and has qualitative differences compared to incoherent TT formation through an avoided crossing. Hence intermediate polarity solvents provide an optimal regime for singlet fission, qualitatively reproducing the experimental trend for the singlet fission rates. The different singlet fission regimes are summarized in the multidimensional plot of Figure 7 for the $\mathrm{CT}_{\uparrow}$ and $\mathrm{TT}$ surfaces. For the different dihedral angles and solvent dielectric constants, the surfaces are annotated with the respective $\mathrm{CT} / \mathrm{TT}$ contribution as a color code.

\section{Conclusions}

Our results demonstrate not only that intramolecular singlet fission in DT-Mes is a 'hot' process mediated by an 
unusual 'destabilized' CT state, but also that the system can explore coherent and incoherent regimes of singlet fission. This is achieved by exploiting symmetry-breaking solvent interactions to induce the necessary energetic proximity for a vibrational mode to mix the $\mathrm{CT}$ and TT states. Interestingly, these interactions are by their nature transient, driven by changes in the solvation shell. Here, the precise degree of mixing depends on the solvent polarity, allowing us to switch between coherent and incoherent fission in different solvents and explore the effects in detail through altering the energy of excitation. In addition, while coherent singlet fission has been

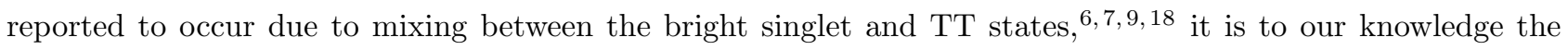
first time that the role of $\mathrm{CT} / \mathrm{TT}$ mixing for coherent fission is studied in detail. The fact that singlet fission in DT-Mes occurs through a real CT intermediate underlines the importance of this second step of fission and the advantages of a system dependent on CT states, which are markedly easier to control through environmental factors such as solvent polarity than LE states.

Our approach for controlling the fission mechanism also indicates a more general concept, where various symmetry-breaking effects could be used as 'switches' between coherent and incoherent regimes. External electric fields and strong coupling to light ${ }^{79}$ could potentially provide a similar symmetry-breaking effect on the CT states, leading to a coherent fission regime in the vicinity of large mixing, as visualized in Figure 8, The same could be achieved through chemical synthesis, or through changes in the crystal symmetry. Experiments 10 and calculations ${ }^{80}$ indicate that the rate of fission closely depends on the crystal structure. Tetracene and TIPStetracene exhibit strikingly different fission properties for highly crystalline versus symmetry-broken amorphous or polycrystalline films 10$] 16] 81$ indicating a possible transition between coherent and incoherent fission within the same material. Singlet fission is but one example of a photophysical process where the efficiency may be tuned by entering a coherent regime. The same underlying principle could also be used to manipulate and ultimately enhance processes as diverse as long-range energy transport, biomimetic light harvesting and charge separation at interfaces in solar cells $82[83$

\section{Methods}

\section{Computations}

In order to obtain the energies of the ground state at the different dihedral angles $\Phi$, constrained geometry optimizations along $\Phi$ were performed using density functional theory (DFT), employing the cc-pVDZ basis set and the B3LYP functional. The DFT calculations allow us to take steric effects into account. To accurately account for conjugation effects on the excited states, excited state calculations at all geometries were performed by using the Pople-Parr-Pariser (PPP) theory. Details on the used PPP parameters and the rest of the computational methodology are given in SI Section S1. The results on the electronic structure and geometries are used to define a model Hamiltonian as outlined in SI Section S1.2. This includes the effect of the dihedral rotation on the electronic structure, as well as that of the solvent through the control parameter $\Delta$. By diagonalizing the Hamiltonian at different dihedral angles, we obtain the wavefunctions and energies of the system. 


\section{Acknowledgements}

This work was supported by the Engineering and Physical Sciences Research Council, UK (grant numbers EP/L015552/1, EP/M025330/1 and EP/M005143/1). A.M.A. acknowledges the support of the Winton Programme for the Physics of Sustainability. S.L. thanks A*STAR Graduate Scholarship support from A*STAR Singapore. T.J.H.H. acknowledges a Research Fellowship from Jesus College, Cambridge. E.G.F. acknowledges financial support from the National Science Foundation Award No. CHE-1555205. J. W. acknowledges financial support from the MOE Tier 3 programme (MOE2014-T3-1-004). The authors thank Akshay Rao for useful discussions and for commenting on the manuscript.

\section{Data Availability}

The data underlying this publication is available at [URL added in proof].

\section{Additional Information}

Supporting Information. Details on the computational methodology, synthesis, and experimental setup and procedure can be found at [URL added in proof].

Competing interests: The authors declare no competing interests

\section{References}

${ }^{1}$ Millicent B. Smith and Josef Michl. Singlet fission. Chemical Reviews, 110(11):6891-6936, 2010.

${ }^{2}$ M. C. Hanna and A. J. Nozik. Solar conversion efficiency of photovoltaic and photoelectrolysis cells with carrier multiplication absorbers. Journal of Applied Physics, 100(7), 2006.

${ }^{3}$ Akshay Rao and Richard H Friend. Harnessing singlet exciton fission to break the Shockley-Queisser limit. Nature Reviews Materials, 2:17063, oct 2017 .

${ }^{4}$ DN Congreve, Jiye Lee, NJ Thompson, and Eric Hontz. External Quantum Efficiency Above 100\% in a Singlet-ExcitonFission-Based Organic Photovoltaic Cell. Science, 340(6130):334-337, 2013.

${ }^{5}$ Wai Lun Chan, Manuel Ligges, Askat E. Jailaubekov, Loren G. Kaake, Luis Miaja-Avila, and X.-Y. Zhu. Observing the Multiexciton State in Singlet Fission and Ensuing Ultrafast Multielectron Transfer. Science, 334(December):1541-1545, 2011.

${ }^{6}$ Wai Lun Chan, Timothy C. Berkelbach, Makenzie R. Provorse, Nicholas R. Monahan, John R. Tritsch, Mark S. Hybertsen, David R. Reichman, Jiali Gao, and X. Y. Zhu. The quantum coherent mechanism for singlet fission: Experiment and theory. Accounts of Chemical Research, 46(6):1321-1329, 2013.

${ }^{7}$ Eric C. Greyson, Josh Vura-Weis, Josef Michl, and Mark A. Ratner. Maximizing singlet fission in organic dimers: Theoretical investigation of triplet yield in the regime of localized excitation and fast coherent electron transfer. Journal of Physical Chemistry $B, 114(45): 14168-14177,2010$.

${ }^{8}$ Nicholas R. Monahan, Dezheng Sun, Hiroyuki Tamura, Kristopher W. Williams, Bolei Xu, Yu Zhong, Bharat Kumar, Colin Nuckolls, Avetik R. Harutyunyan, Gugang Chen, Hai Lung Dai, David Beljonne, Yi Rao, and X. Y. Zhu. Dynamics of the triplet-pair state reveals the likely coexistence of coherent and incoherent singlet fission in crystalline hexacene. Nature Chemistry, 9(4):341-346, 2017.

${ }^{9}$ Kiyoshi Miyata, Yuki Kurashige, Kazuya Watanabe, Toshiki Sugimoto, Shota Takahashi, Shunsuke Tanaka, Jun Takeya, Takeshi Yanai, and Yoshiyasu Matsumoto. Coherent singlet fission activated by symmetry breaking. Nature Chemistry, 9(10):983-989, 2017. 

Congreve, Akshay Rao, Kerr Johnson, Matthew Y Sfeir, Moungi G Bawendi, Timothy M Swager, Richard H Friend, Marc a Baldo, and Troy Van Voorhis. A transferable model for singlet-fission kinetics. Nature Chemistry, 6(6):492-497, 2014.

${ }^{11}$ Paul M Zimmerman, Zhiyong Zhang, and Charles B Musgrave. Singlet fission in pentacene through multi-exciton quantum states. Nature chemistry, 2(8):648-652, 2010.

12 C. E. Swenberg and W. T. Stacy. Bimolecular radiationless transitions in crystalline tetracene. Chemical Physics Letters, $2(5): 327-328,1968$.

13 Jonathan J. Burdett and Christopher J. Bardeen. Quantum beats in crystalline tetracene delayed fluorescence due to triplet pair coherences produced by direct singlet fission. Journal of the American Chemical Society, 134(20):8597-8607, 2012.

${ }^{14}$ Mark W.B. Wilson, Akshay Rao, Kerr Johnson, Simon Gélinas, Riccardo Di Pietro, Jenny Clark, and Richard H. Friend. Temperature-independent singlet exciton fission in tetracene. Journal of the American Chemical Society, 135(44):16680-16688, 2013.

${ }^{15}$ Murad J.Y. Tayebjee, Raphaël G.C.R. Clady, and Timothy W. Schmidt. The exciton dynamics in tetracene thin films. Physical Chemistry Chemical Physics, 15(35):14797-14805, 2013.

${ }^{16}$ Hannah L. Stern, Alexandre Cheminal, Shane R. Yost, Katharina Broch, Sam L. Bayliss, Kai Chen, Maxim Tabachnyk, Karl Thorley, Neil Greenham, Justin M. Hodgkiss, John Anthony, Martin Head-Gordon, Andrew J. Musser, Akshay Rao, and Richard H. Friend. Vibronically coherent ultrafast triplet-pair formation and subsequent thermally activated dissociation control efficient endothermic singlet fission. Nature Chemistry, 9(12):1205-1212, 2017.

17 Jonathan J. Burdett, David Gosztola, and Christopher J. Bardeen. The dependence of singlet exciton relaxation on excitation density and temperature in polycrystalline tetracene thin films: Kinetic evidence for a dark intermediate state and implications for singlet fission. Journal of Chemical Physics, 135(21), 2011.

${ }^{18}$ Wai Lun Chan, Manuel Ligges, and X. Y. Zhu. The energy barrier in singlet fission can be overcome through coherent coupling and entropic gain. Nature Chemistry, 4(10):840-845, 2012.

19 Andrew J Musser, Matz Liebel, Christoph Schnedermann, Torsten Wende, Tom B Kehoe, Akshay Rao, and Philipp Kukura. Evidence for conical intersection dynamics mediating ultrafast singlet exciton fission. Nature Physics, 11:352, mar 2015.

${ }^{20}$ Artem A. Bakulin, Sarah E. Morgan, Tom B. Kehoe, Mark W.B. Wilson, Alex W. Chin, Donatas Zigmantas, Dassia Egorova, and Akshay Rao. Real-time observation of multiexcitonic states in ultrafast singlet fission using coherent $2 \mathrm{D}$ electronic spectroscopy. Nature Chemistry, 8(1):16-23, 2016.

${ }^{21}$ Roel Tempelaar and David R. Reichman. Vibronic exciton theory of singlet fission. III. How vibronic coupling and thermodynamics promote rapid triplet generation in pentacene crystals. Journal of Chemical Physics, 148(24), 2018.

${ }^{22}$ Yuta Fujihashi, Lipeng Chen, Akihito Ishizaki, Junling Wang, and Yang Zhao. Effect of high-frequency modes on singlet fission dynamics. Journal of Chemical Physics, 146(4), 2017.

${ }^{23}$ Hiroyuki Tamura, Miquel Huix-Rotllant, Irene Burghardt, Yoann Olivier, and David Beljonne. First-Principles Quantum Dynamics of Singlet Fission: Coherent versus Thermally Activated Mechanisms Governed by Molecular $\pi$ Stacking. Physical Review Letters, 115(10):1-5, 2015.

${ }^{24}$ Xintian Feng and Anna I. Krylov. On couplings and excimers: lessons from studies of singlet fission in covalently linked tetracene dimers. Physical Chemistry Chemical Physics, 18(11):7751-7761, 2016.

25 Zhongkai Huang, Yuta Fujihashi, and Yang Zhao. Effect of Off-Diagonal Exciton-Phonon Coupling on Intramolecular Singlet Fission. The Journal of Physical Chemistry Letters, 8(14):3306-3312, jul 2017.

${ }^{26}$ Florian A. Y. N. Schröder, David H. P. Turban, Andrew J. Musser, Nicholas D. M. Hine, and Alex W. Chin. Tensor network simulation of multi-environmental open quantum dynamics via machine learning and entanglement renormalisation. Nature Communications, 10(1):1062, 2019. 

Greenham, and Andrew J. Musser. Tuning the role of charge-transfer states in intramolecular singlet exciton fission through side-group engineering. Nature Communications, 7:13622, 2016.

${ }^{28}$ Eric G. Fuemmeler, Samuel N. Sanders, Andrew B. Pun, Elango Kumarasamy, Tao Zeng, Kiyoshi Miyata, Michael L. Steigerwald, X. Y. Zhu, Matthew Y. Sfeir, Luis M. Campos, and Nandini Ananth. A direct mechanism of ultrafast intramolecular singlet fission in pentacene dimers. ACS Central Science, 2(5):316-324, 2016.

29 Timothy C. Berkelbach, Mark S. Hybertsen, and David R. Reichman. Microscopic theory of singlet exciton fission. II. Application to pentacene dimers and the role of superexchange. Journal of Chemical Physics, 138(11):114103, 2013.

${ }^{30}$ Paul M. Zimmerman, Franziska Bell, David Casanova, and Martin Head-Gordon. Mechanism for singlet fission in pentacene and tetracene: From single exciton to two triplets. Journal of the American Chemical Society, 133(49):19944-19952, 2011.

${ }^{31}$ Millicent B. Smith and Josef Michl. Recent Advances in Singlet Fission. Annual Review of Physical Chemistry, 64(1):361-386, 2013.

32 Timothy C. Berkelbach, Mark S. Hybertsen, and David R. Reichman. Microscopic theory of singlet exciton fission. I. General formulation. The Journal of Chemical Physics, 138(11):114102, 2013.

${ }^{33}$ K. C. Krishnapriya, Palas Roy, Boregowda Puttaraju, Ulrike Salzner, Andrew J. Musser, Manish Jain, Jyotishman Dasgupta, and Satish Patil. Spin density encodes intramolecular singlet exciton fission in pentacene dimers. Nature Communications, 10(1), 2019 .

34 Samuel N. Sanders, Elango Kumarasamy, Andrew B. Pun, M. Tuan Trinh, Bonnie Choi, Jianlong Xia, Elliot J. Taffet, Jonathan Z. Low, John R. Miller, Xavier Roy, X. Y. Zhu, Michael L. Steigerwald, Matthew Y. Sfeir, and Luis M. Campos. Quantitative Intramolecular Singlet Fission in Bipentacenes. Journal of the American Chemical Society, 137(28):8965-8972, 2015.

35 Johannes Zirzlmeier, Dan Lehnherr, Pedro B. Coto, Erin T. Chernick, Rubén Casillas, Bettina S. Basel, Michael Thoss, Rik R. Tykwinski, and Dirk M. Guldi. Singlet fission in pentacene dimers. Proceedings of the National Academy of Sciences, $112(17): 5325-5330,2015$.

36 Nadezhda V. Korovina, Saptaparna Das, Zachary Nett, Xintian Feng, Jimmy Joy, Ralf Haiges, Anna I. Krylov, Stephen E. Bradforth, and Mark E. Thompson. Singlet Fission in a Covalently Linked Cofacial Alkynyltetracene Dimer. Journal of the American Chemical Society, 138(2):617-627, 2016.

37 Justin C. Johnson, Arthur J. Nozik, and Josef Michl. The role of chromophore coupling in singlet fission. Accounts of Chemical Research, 46(6):1290-1299, 2013.

38 Elango Kumarasamy, Samuel N. Sanders, Murad J.Y. Tayebjee, Amir Asadpoordarvish, Timothy J.H. Hele, Eric G. Fuemmeler, Andrew B. Pun, Lauren M. Yablon, Jonathan Z. Low, Daniel W. Paley, Jacob C. Dean, Bonnie Choi, Gregory D. Scholes, Michael L. Steigerwald, Nandini Ananth, Dane R. McCamey, Matthew Y. Sfeir, and Luis M. Campos. Tuning Singlet Fission in $\pi$-Bridge- $\pi$ Chromophores. Journal of the American Chemical Society, 139(36):12488-12494, 2017.

39 Bettina S Basel, Johannes Zirzlmeier, Constantin Hetzer, Brian T Phelan, Matthew D Krzyaniak, S Rajagopala Reddy, Pedro B Coto, Noah E Horwitz, Ryan M Young, Fraser J White, Frank Hampel, Timothy Clark, Michael Thoss, Rik R Tykwinski, Michael R Wasielewski, and Dirk M Guldi. Unified model for singlet fission within a non-conjugated covalent pentacene dimer. Nature Communications, 8(May):15171, may 2017.

40 Jasper D Cook, Thomas James Carey, Dylan H. Arias, Justin C. Johnson, and Niels H. Damrauer. Solvent-Controlled Branching of Localized versus Delocalized Singlet Exciton States and Equilibration with Charge Transfer in a Structurally Well-Defined Tetracene Dimer. The Journal of Physical Chemistry A, 121(48):9229-9242, dec 2017.

${ }^{41}$ Nadezhda V. Korovina, Jimmy Joy, Xintian Feng, Cassidy Feltenberger, Anna I. Krylov, Stephen E. Bradforth, and Mark E. Thompson. Linker-Dependent Singlet Fission in Tetracene Dimers. Journal of the American Chemical Society, 140(32):1017910190, 2018. 
42 Takuya Yamakado, Shota Takahashi, Kazuya Watanabe, Yoshiyasu Matsumoto, Atsuhiro Osuka, and Shohei Saito. Conformational Planarization versus Singlet Fission: Distinct Excited-State Dynamics of Cyclooctatetraene-Fused Acene Dimers. Angewandte Chemie International Edition, 57(19):5438-5443, may 2018.

43 Takao Sakuma, Hayato Sakai, Yasuyuki Araki, Tadashi Mori, Takehiko Wada, Nikolai V. Tkachenko, and Taku Hasobe. LongLived Triplet Excited States of Bent-Shaped Pentacene Dimers by Intramolecular Singlet Fission. Journal of Physical Chemistry A, 120(11):1867-1875, 2016.

${ }^{44}$ Steven Lukman, Andrew J. Musser, Kai Chen, Stavros Athanasopoulos, Chaw K. Yong, Zebing Zeng, Qun Ye, Chunyan Chi, Justin M. Hodgkiss, Jishan Wu, Richard H. Friend, and Neil C. Greenham. Tuneable Singlet Exciton Fission and Triplet-Triplet Annihilation in an Orthogonal Pentacene Dimer. Advanced Functional Materials, 25(34):5452-5461, 2015.

${ }^{45}$ Eric A. Margulies, Claire E. Miller, Yilei Wu, Lin Ma, George C. Schatz, Ryan M. Young, and Michael R. Wasielewski. Enabling singlet fission by controlling intramolecular charge transfer in $\pi$-stacked covalent terrylenediimide dimers. Nature Chemistry, 8(12):1120-1125, 2016.

${ }^{46}$ Andrew J. Musser, Mohammed Al-Hashimi, Margherita Maiuri, Daniele Brida, Martin Heeney, Giulio Cerullo, Richard H. Friend, and Jenny Clark. Activated singlet exciton fission in a semiconducting polymer. Journal of the American Chemical Society, 135(34):12747-12754, 2013.

${ }^{47}$ Karsten Elich, Minoru Kitazawa, Tadashi Okada, and Rüdiger Wortmann. Effect of S 1 Torsional Dynamics on the Time-Resolved Fluorescence Spectra of 9,9'-Bianthryl in Solution. The Journal of Physical Chemistry A, 101(96):2010-2015, 1997.

${ }^{48}$ Yuxiang Mo, Jiangtao Lei, Yunxiang Sun, Qingwen Zhang, and Guanghong Wei. Conformational Ensemble of hIAPP Dimer: Insight into the Molecular Mechanism by which a Green Tea Extract inhibits hIAPP Aggregation. Scientific Reports, 6(May):111, 2016.

${ }^{49}$ C. A. Coulson and G. S. Rushbrooke. Note on the method of molecular orbitals. Mathematical Proceedings of the Cambridge Philosophical Society, 36(2):193-200, 1940.

${ }^{50}$ N. J. Hestand, H. Yamagata, Bolei Xu, Dezheng Sun, Yu Zhong, Avetik R. Harutyunyan, Gugang Chen, Hai Lung Dai, Yi Rao, and F. C. Spano. Polarized absorption in crystalline pentacene: Theory vs experiment. Journal of Physical Chemistry C, 119(38):22137-22147, 2015.

${ }^{51} \mathrm{G}$. Wilse Robinson. Intensity enhancement of forbidden electronic transitions by weak intermolecular interactions. The Journal of Chemical Physics, 46(2):572-585, 1967.

52 Annamaria Petrozza, Frédéric Laquai, Ian A. Howard, Ji-Seon Kim, and Richard H. Friend. Dielectric switching of the nature of excited singlet state in a donor-acceptor-type polyfluorene copolymer. Phys. Rev. B, 81:205421, May 2010.

${ }^{53}$ Shunichi Fukuzumi, Hiroaki Kotani, Kei Ohkubo, Seiji Ogo, Nikolai V. Tkachenko, and Helge Lemmetyinen. Electron-Transfer State of 9-Mesityl-10-methylacridinium Ion with a Much Longer Lifetime and Higher Energy Than That of the Natural Photosynthetic Reaction Center. Journal of the American Chemical Society, 126(6):1600-1601, 2004.

${ }^{54}$ Samuel W. Eaton, Leah E. Shoer, Steven D. Karlen, Scott M. Dyar, Eric A. Margulies, Brad S. Veldkamp, Charusheela Ramanan, Daniel A. Hartzler, Sergei Savikhin, Tobin J. Marks, and Michael R. Wasielewski. Singlet exciton fission in polycrystalline thin films of a slip-stacked perylenediimide. Journal of the American Chemical Society, 135(39):14701-14712, 2013.

${ }^{55}$ Hannah L. Stern, Andrew J. Musser, Simon Gelinas, Patrick Parkinson, Laura M. Herz, Matthew J. Bruzek, John Anthony, Richard H. Friend, and Brian J. Walker. Identification of a triplet pair intermediate in singlet exciton fission in solution. Proceedings of the National Academy of Sciences, 112(25):7656-7661, 2015.

${ }^{56}$ Hiroki Nagashima, Shuhei Kawaoka, Seiji Akimoto, Takashi Tachikawa, Yasunori Matsui, Hiroshi Ikeda, and Yasuhiro Kobori. Singlet-Fission-Born Quintet State: Sublevel Selections and Trapping by Multiexciton Thermodynamics. Journal of Physical Chemistry Letters, 9(19):5855-5861, 2018.

${ }^{57}$ Kai Chen, Joseph K. Gallaher, Alex J. Barker, and Justin M. Hodgkiss. Transient grating photoluminescence spectroscopy: An ultrafast method of gating broadband spectra. Journal of Physical Chemistry Letters, 5(10):1732-1737, 2014. 
${ }^{58}$ Martin Jurczok, Pascal Plaza, Monique M. Martin, Yves H. Meyer, and Wolfgang Rettig. Excited state relaxation paths in 9,9'-bianthryl and 9-carbazolyl-anthracene: A sub-ps transient absorption study. Chemical Physics, 253(2-3):339-349, 2000.

${ }^{59}$ C. Burgdorff, T. Kircher, and H. G. Löhmannsröben. Photophysical properties of tetracene derivatives in solution. Spectrochimica Acta Part A: Molecular Spectroscopy, 44(11):1137-1141, 1988.

60 Akshay Rao, Philip C Y Chow, Simon Gélinas, Cody W Schlenker, Chang-Zhi Li, Hin-Lap Yip, Alex K-Y Jen, David S Ginger, and Richard H Friend. The role of spin in the kinetic control of recombination in organic photovoltaics. Nature, 500(7463):435-9, 2013.

${ }^{61}$ Samuel N. Sanders, Elango Kumarasamy, Andrew B. Pun, Michael L. Steigerwald, Matthew Y. Sfeir, and Luis M. Campos. Intramolecular Singlet Fission in Oligoacene Heterodimers. Angewandte Chemie - International Edition, 55(10):3373-3377, 2016.

${ }^{62}$ Murad J.Y. Tayebjee, Samuel N. Sanders, Elango Kumarasamy, Luis M. Campos, Matthew Y. Sfeir, and Dane R. McCamey. Quintet multiexciton dynamics in singlet fission. Nature Physics, 13(2):182-188, 2017.

${ }^{63}$ Erik Busby, Jianlong Xia, Qin Wu, Jonathan Z. Low, Rui Song, John R. Miller, X. Y. Zhu, Luis M. Campos, and Matthew Y. Sfeir. A design strategy for intramolecular singlet fission mediated by charge-transfer states in donor-acceptor organic materials. Nature Materials, 14(4):426-433, 2015.

${ }^{64}$ Yukitomo Kasai, Yasunari Tamai, Hideo Ohkita, Hiroaki Benten, and Shinzaburo Ito. Ultrafast Singlet Fission in a Push-Pull Low-Bandgap Polymer Film. Journal of the American Chemical Society, 137(51):15980-15983, 2015.

${ }^{65}$ Anatoly B. Kolomeisky, Xintian Feng, and Anna I. Krylov. A simple kinetic model for singlet fission: A role of electronic and entropic contributions to macroscopic rates. Journal of Physical Chemistry C, 118(10):5188-5195, 2014.

${ }^{66}$ Chaw Keong Yong, Andrew J. Musser, Sam L. Bayliss, Steven Lukman, Hiroyuki Tamura, Olga Bubnova, Rawad K. Hallani, Aurelie Meneau, Roland Resel, Munetaka Maruyama, Shu Hotta, Laura M. Herz, David Beljonne, John E. Anthony, Jenny Clark, and Henning Sirringhaus. The entangled triplet pair state in acene and heteroacene materials. Nature Communications, 8(May):15953, 2017.

${ }^{67}$ Steven Lukman, Johannes M. Richter, Le Yang, Pan Hu, Jishan Wu, Neil C. Greenham, and Andrew J. Musser. Efficient Singlet Fission and Triplet-Pair Emission in a Family of Zethrene Diradicaloids. Journal of the American Chemical Society, 139(50):18376-18385, 2017.

68 Andrew J. Musser and Jenny Clark. Triplet-pair states in organic semiconductors. Annual Review of Physical Chemistry, 70:323-351, 2019.

${ }^{69}$ Xintian Feng, Anatoliy V. Luzanov, and Anna I. Krylov. Fission of entangled Spins: An electronic structure perspective. Journal of Physical Chemistry Letters, 4(22):3845-3852, 2013.

${ }^{70}$ Clark Robin J H and Trevor J Dines. Resonance Raman Spectroscopy, and Its Application to Inorganic Chemistry. Angewandte Chemie - International Edition, 25:131-158, 1986.

${ }^{71}$ H. Köppel, Wolfgang Domcke, and Lorenz S Cederbaum. Advances in Chemical Physics, Volume 57, Multimode Molecular Dynamics Beyond the Born-Oppenheimer Approximation, pages 102-110. 1984.

${ }^{72}$ Fabrizia Negri and G. Orlandi. Computational Photochemistry, Chapter IV, pages 129-139. Elsevier, 2005.

${ }^{73}$ Xin Tan, Terry L. Gustafson, Christophe Lefumeux, Gotard Burdzinski, Guy Buntinx, and Olivier Poizat. Solvation dynamics probed by femtosecond transient absorption spectroscopy: Vibrational cooling and conformational relaxation in S1 trans-4,4diphenylstibene. Journal of Physical Chemistry A, 106(14):3593-3598, 2002.

${ }^{74}$ A. Weigel and N. P. Ernsting. Excited Stilbene: Intramolecular Vibrational Redistribution and Solvation Studied by Femtosecond Stimulated Raman Spectroscopy. Journal of Physical Chemistry B, 114(23):7879-7893, 2010.

${ }^{75}$ Michael P. Grubb, Philip M. Coulter, Hugo J.B. Marroux, Balazs Hornung, Ryan S. McMullen, Andrew J. Orr-Ewing, and Michael N.R. Ashfold. Translational, rotational and vibrational relaxation dynamics of a solute molecule in a non-interacting solvent. Nature Chemistry, 8(11):1042-1046, 2016. 


\footnotetext{
${ }^{76}$ Michael P. Grubb, Philip M. Coulter, Hugo J.B. Marroux, Andrew J. Orr-Ewing, and Michael N.R. Ashfold. Unravelling the mechanisms of vibrational relaxation in solution. Chemical Science, 8(4):3062-3069, 2017.

77 Timothy John Harvey Hele, Eric G Fuemmeler, Samuel N Sanders, Elango Kumarasamy, Matthew Y. Sfeir, Luis M. Campos, and Nandini Ananth. Anticipating Acene-based Chromophore Spectra with Molecular Orbital Arguments. The Journal of Physical Chemistry A, 123:2527-2536, 2019.

${ }^{78}$ W. M. Kwok, C. Ma, M. W. George, D. C. Grills, P. Matousek, A. W. Parker, D. Phillips, W. T. Toner, and M. Towrie. Solvent effects on the charge transfer excited states of 4-dimethylaminobenzonitrile (DMABN) and 4-dimethylamino-3,5dimethylbenzonitrile (TMABN) studied by time-resolved infrared spectroscopy: a direct observation of hydrogen bonding interactions. Photochemical \& Photobiological Sciences, 6(9):987, 2007.

${ }^{79}$ Daniel Polak, Rahul Jayaprakash, Anastasia Leventis, Kealan J Fallon, Harriet Coulthard, Anthony J. Petty, John Anthony, Hugo Bronstein, David G Lidzey, Jenny Clark, and Andrew J Musser. Manipulating matter with strong coupling: harvesting triplet excitons in organic exciton microcavities. arXiv, page 1806.09990, 2018.

80 Sivan Refaely-Abramson, Felipe H. Da Jornada, Steven G. Louie, and Jeffrey B. Neaton. Origins of Singlet Fission in Solid Pentacene from an ab initio Green's Function Approach. Physical Review Letters, 119(26):1-6, 2017.

${ }^{81}$ Geoffrey B. Piland and Christopher J. Bardeen. How Morphology Affects Singlet Fission in Crystalline Tetracene. Journal of Physical Chemistry Letters, 6(10):1841-1846, 2015.

82 Simon Gélinas, Akshay Rao, Abhishek Kumar, Samuel L Smith, Alex W Chin, Jenny Clark, Tom S van der Poll, Guillermo C Bazan, and Richard H Friend. Ultrafast long-range charge separation in organic semiconductor photovoltaic diodes. Science (New York, N.Y.), 343(6170):512-6, 2014.

83 Gregory D. Scholes, Graham R. Fleming, Lin X. Chen, Alán Aspuru-Guzik, Andreas Buchleitner, David F. Coker, Gregory S. Engel, Rienk Van Grondelle, Akihito Ishizaki, David M. Jonas, Jeff S. Lundeen, James K. McCusker, Shaul Mukamel, Jennifer P. Ogilvie, Alexandra Olaya-Castro, Mark A. Ratner, Frank C. Spano, K. Birgitta Whaley, and Xiaoyang Zhu. Using coherence to enhance function in chemical and biophysical systems. Nature, 543(7647):647-656, 2017.
} 


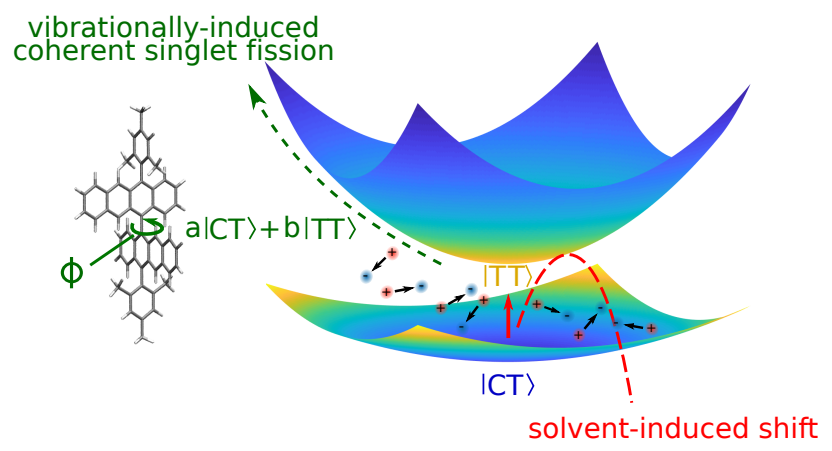

For Table of Contents Only 